\title{
Modulation of Premotor and Primary Motor Cortical Activity during Volitional Adjustments of Speed-Accuracy Trade-Offs
}

\author{
David Thura and Paul Cisek \\ Groupe de Recherche sur le Système Nerveux Central, Département de neurosciences, Université de Montréal, Montréal, Québec H3T 1J4, Canada
}

Recent work suggests that while animals decide between reaching actions, neurons in dorsal premotor (PMd) and primary motor (M1) cortex reflect a dynamic competition between motor plans and determine when commitment to a choice is made. This competition is biased by at least two sources of information: the changing sensory evidence for one choice versus another, and an urgency signal that grows over time. Here, we test the hypothesis that the urgency signal adjusts the trade-off between speed and accuracy during both decision-making and movement execution. Two monkeys performed a reaching decision task in which sensory evidence continuously evolves over the course of each trial. In different blocks, task timing parameters encouraged monkeys to voluntarily adapt their behavior to be either hasty or conservative. Consistent with our hypothesis, during the deliberation process the baseline and gain of neural activity in decision-related PMd (29\%) and M1 cells (45\%) was higher when monkeys applied a hasty policy than when they behaved conservatively, but at the time of commitment the population activity was similar across blocks. Other cells ( $30 \%$ in PMd, $30 \%$ in M1) showed activity that increased or decreased with elapsing time until the moment of commitment. Movement-related neurons were also more active after longer decisions, as if they were influenced by the same urgency signal controlling the gain of decision-related activity. Together, these results suggest that the arm motor system receives an urgency/vigor signal that adjusts the speed-accuracy trade-off for decision-making and movement execution.

Key words: decision-making; monkey; speed-accuracy trade-off; urgency

\section{Significance Statement}

This work addresses the neural mechanisms that control the speed-accuracy trade-off in both decisions and movements, in the kinds of dynamic situations that are typical of natural animal behavior. We found that many "decision-related" premotor and motor neurons are modulated in a time-dependent manner compatible with an "urgency" signal that changes between hasty and conservative decision policies. We also found that such modulation influenced cells related to the speed of the reaching movements executed by the animals to report their decisions. These results suggest that a unified mechanism determines speedaccuracy trade-off adjustments during decision-making and movement execution, potentially influencing both the cognitive and motor aspects of reward-oriented behavior.

\section{Introduction}

During natural behavior, animals are motivated to optimize their reward rate. To do so, they must find the best speed-accuracy

\footnotetext{
Received June 9, 2015; revised Nov. 26, 2015; accepted Dec. 9, 2015.

Author contributions: P.C. designed research; D.T. performed research; D.T. and P.C. analyzed data; D.T. and P.C. wrote the paper.

This work was supported by Canadian Institutes of Health Research Grant MOP-102662, the Canadian Foundation for Innovation, Fonds de Recherche en Santé du Québec, the EJLB Foundation to P.C., and fellowships from the FYSSEN Foundation and the Groupe de Recherche sur le Système Nerveux Central to D.T. We thank Marie-Claude Labonté for technical support.

The authors declare no competing financial interests.

Correspondence should be addressed to Dr. David Thura, Université de Montréal, 2960 Chemin de la Tour,

Montréal, Québec H3T 1J4, Canada. E-mail: david.thura@umontreal.ca.
}

trade-off (SAT) for both their decisions and their movements, and to adjust it to the current context. Recent studies examined how the SAT is adjusted during a variety of perceptual discrimination tasks. For example, several fMRI studies reported that time pressure leads to an increased BOLD response during baseline periods (Forstmann et al., 2008; Ivanoff et al., 2008; van Veen et al., 2008). Additional mechanisms for SAT adjustment have been identified at the level of single neurons. In the frontal eye fields (FEFs), Heitz and Schall (2012) found baseline changes as well as changes in neural gain and the onset time of perceptual 
processing. Baseline and gain changes were also reported by Hanks et al. (2014) in the lateral intraparietal area. These results are consistent with the proposal that SAT adjustment varies the baseline and drift rate parameters of evidence accumulation models (EAMs) (Bogacz et al., 2010a; Heitz, 2014; Standage et al., 2014).

All of these previous studies used perceptual discrimination tasks in which sensory information is constant within each trial. However, during natural behavior, animals are faced with a dynamic and continuously changing environment; and in such conditions, gradual accumulation of evidence is too sluggish to quickly respond to stimulus changes. Indeed, we recently found that, during deliberation in a dynamic reach-decision task, neural activity in monkey dorsal premotor (PMd) and primary motor cortex (M1) is not compatible with EAMs because it does not reflect integrated evidence (Thura and Cisek, 2014). Instead, consistent with the urgency-gating model (UGM) (Cisek et al., 2009; Thura et al., 2012), activity in both regions combines rapid estimates of evidence with a growing "urgency signal" related to elapsed time. Nevertheless, the way in which SAT is adjusted in this model may be closely related to what has been observed in prior studies. In particular, because the urgency signal effectively controls the dropping accuracy criterion that defines a subject's SAT policy during decision-making (Churchland et al., 2008; Standage et al., 2014), it could also be used to adjust the SAT when timing parameters of the task are varied in a blockdependent manner. Thura et al. (2014) reported behavioral evidence consistent with this proposal and also found that the same urgency signal that energizes the decision also appears to influence the vigor of the selected action, suggesting a unified mechanism of SAT adjustment for both decisions and actions.

Here, we test this hypothesis at the neural level by comparing activity in PMd and M1 while monkeys perform a dynamic decision task in two contexts: one motivating hasty choices and one motivating more conservative behavior. The UGM predicts that, when decisions are hasty, the urgency signal will increase the baseline and gain of both decision- and movement-related neural activity in the motor system. Some of these results previously appeared in abstract form (Thura and Cisek, 2011, 2012).

\section{Materials and Methods}

Subjects and apparatus. Two male macaque monkeys (Macaca mulatta; Monkey S: 6 years old, $6 \mathrm{~kg}$; Monkey Z: 5 years old, $6 \mathrm{~kg}$ ) were implanted, under anesthesia and aseptic conditions, with a titanium head fixation post and recording chambers. The local animal ethics committee approved surgery, testing procedure, and animal care. Monkeys sat headfixed in a custom primate chair and performed two planar reaching tasks using a vertically oriented cordless stylus whose position was recorded by a digitizing tablet (CalComp, $125 \mathrm{~Hz}$ ). Their nonacting hand (Monkey S: left hand for $\sim 2$ years, then right hand for 6 months; Monnkey Z: right hand for $\sim 1$ year, then left hand) was restrained on an arm rest with Velcro bands. In some sessions, unconstrained eye movements were recorded using an infrared camera (ASL, $120 \mathrm{~Hz}$ ). Stimuli and continuous cursor feedback were projected onto a mirror suspended between the monkey's gaze and the tablet, creating the illusion that they are in the plane of the tablet. Neural activity was recorded from the hemisphere contralateral to the acting hand with 1-4 independently moveable (NAN microdrive) microelectrodes (FHC), and data were acquired with the AlphaLab system (Alpha-Omega Eng).

Behavioral tasks. Monkeys were trained to perform the "tokens" task (Fig. 1A) in which they are presented with one central starting circle (1.75 $\mathrm{cm}$ radius) and two peripheral target circles $(1.75 \mathrm{~cm}$ radius, arranged at $180^{\circ}$ around a $5 \mathrm{~cm}$ radius circle). The monkey begins each trial by placing a handle in the central circle, in which 15 small tokens are randomly arranged. The tokens then begin to jump, one-by-one every 200 ms ("predecision interval"), from the center to one of the two peripheral targets. The monkey's task is to move the handle to the target he believes will ultimately receive the majority of tokens. The monkey is allowed to make the decision as soon as he feels sufficiently confident, and has 500 $\mathrm{ms}$ to bring the cursor into a target after leaving the center. Crucially, when the monkey reaches a target, the remaining tokens move more quickly to their final targets ("postdecision interval," which was either 50 or $150 \mathrm{~ms}$ in separate "fast" and "slow" blocks of trials, respectively). Once all tokens have jumped, visual feedback is provided to the monkey (the chosen target turns green for correct choices or red for error trials) and a drop of fruit juice is delivered for choosing the correct target. A $1500 \mathrm{~ms}$ intertrial interval precedes the following trial.

In both fast and slow blocks, the monkey is thus presented with a trade-off: either wait until the decision can be made with confidence, or guess ahead of time, which may not be as reliable but could yield potential successes more quickly (because of the acceleration of the remaining tokens). The trade-off can be adjusted between the two blocks. In particular, hasty decisions are more advantageous (in terms of reward rate) in the fast blocks than in the slow blocks because guessing quickly allows the monkey to save more time. Importantly, because all task events preceding target acquisition are identical between the two blocks, any differences in neural activity between the two blocks before target acquisition must reflect differences in the strategy voluntarily adopted by the monkeys.

The monkeys were also trained to perform a delayed reach task (usually 30-48 trials per session). In this task, the monkey again begins by placing the cursor in the central circle containing the 15 tokens. Next, one of six peripheral targets is presented $\left(1.75 \mathrm{~cm}\right.$ radius, spaced at $60^{\circ}$ intervals around a $5 \mathrm{~cm}$ radius circle); and after a variable delay $(500 \pm 100$ $\mathrm{ms}$ ), the 15 tokens simultaneously jump into that target. This "GO signal" instructs the monkey to move the handle to the target to receive a drop of juice. This task is used to determine cell tuning as well as the animal's mean reaction time (RT), used as an estimate of the total delays attributable to sensory processing and response initiation.

Dataset. The last stage of monkeys' training in the tokens task involved providing animals with alternating blocks of slow and fast trials $(\sim 100-$ 150 trials in a block) of the tokens task. Based on behavioral data (for a detailed analysis of the same animals' behavior, see Thura et al., 2014), we defined two periods during this last stage: first, when behavior was comparable between the two blocks; and second, when the monkeys began to behave differently in the two blocks, in terms of decision duration (DD) and success probability. Because the main goal of the present study is to explore neural correlates of monkeys' SAT adjustment between the blocks, data presented in this report only include trials performed during this final stage of training.

Neural recordings. Our standard procedures for single-unit recordings in the PMd and M1, signal processing, and data management have been described previously (Thura and Cisek, 2014). During recording sessions, we focused on cells showing a change of activity in the tokens task, and monkeys were usually performing the task while we were searching for cells. When one or more task-related cells were isolated, we ran a block of 30-48 trials of the delayed reach task to determine spatial tuning and select a preferred target (PT) for each cell (i.e., the target associated with the highest firing rate during one or more task epochs). Next, we ran blocks of tokens task trials using the PT of an isolated cell and the $180^{\circ}$ opposite target (OT). We sometimes simultaneously recorded several task-related cells showing different spatial preferences; and because we always selected a single pair of targets, the actual best direction for each of the recorded cells was not always among these two.

We usually started recording cells in the slow block because monkeys were more conservative in this condition (see Results). It was thus easier to assess cell properties online and more convenient to search for cells because fewer rewards were spent. When possible, cells were tested with multiple repetitions of slow and fast blocks to control for potential confounds related to evolving signals, elapsing time, and the monkey's fatigue or satiation.

Behavioral data analysis. Methods to analyze monkeys' behavior in the tokens task have been described previously (Cisek et al., 2009; Thura and Cisek, 2014). Briefly, the tokens task allows us to calculate, at each moment in time, the "success probability" associated with choosing each target. To characterize the success probability profile for each trial, we 
A

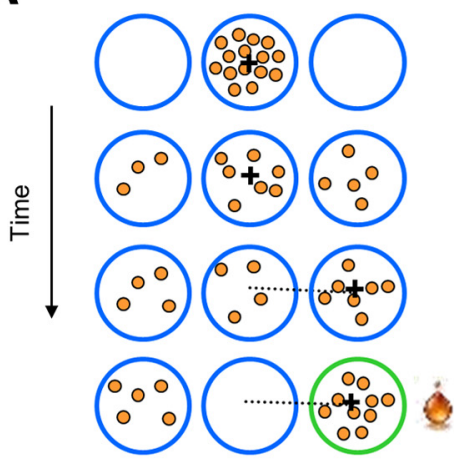

B

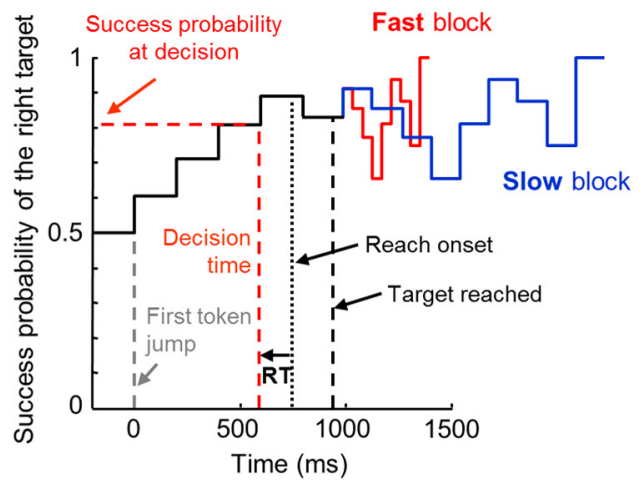

C

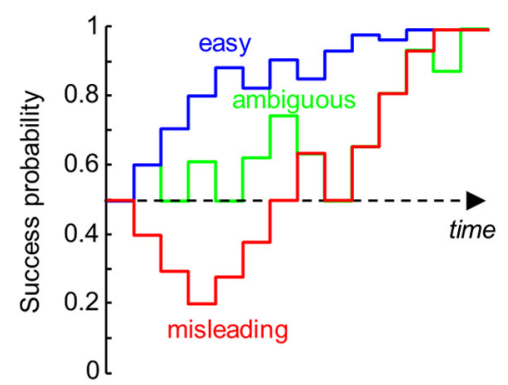

D

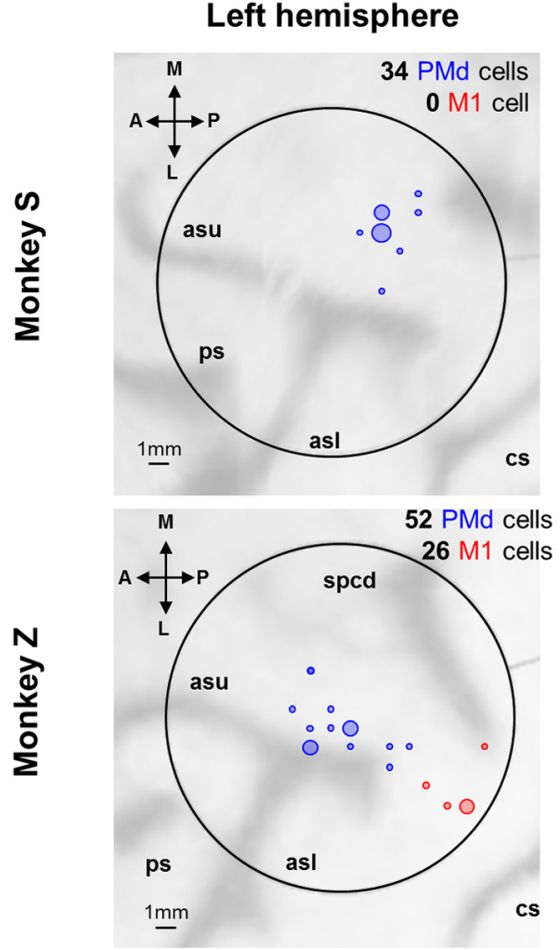

\section{Right hemisphere}
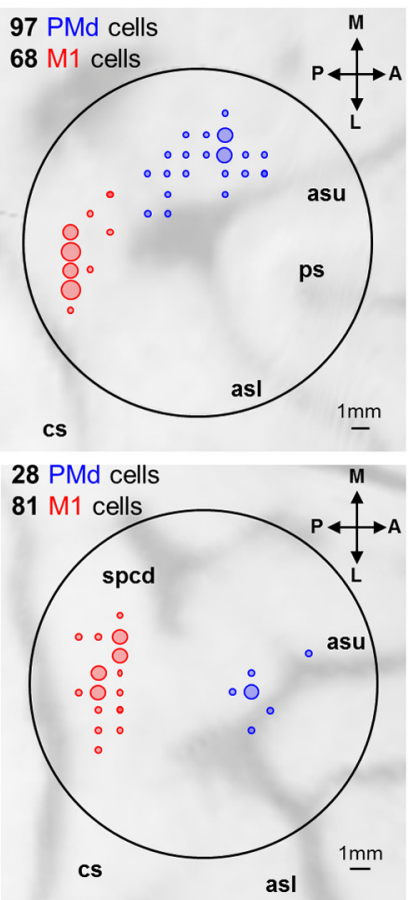

ps : principal sulcus

spcd: superior precentral dimple asu: arcuate sulcus upper limb asl: arcuate sulcus lower limb cs: central sulcus

Cells classified as PMd cells

Cells classified as M1 cells

Figure 1. A, The tokens task. Each row illustrates a step in an example trial. $\boldsymbol{B}$, Success probability profile of an example trial. Before the chosen target is reached, tokens jump every 200 ms into one of the two potential targets. The probability of success of choosing each target therefore evolves over time (black trace, for the right target in this example). After the chosen target is reached (vertical black dashed line), the remaining tokens either jump every $150 \mathrm{~ms}$ (in slow blocks, blue trace) or every $50 \mathrm{~ms}$ (fast block, red trace) to their allocated target. We detect the time of movement onset (vertical black dotted line) and subtract from it the mean RT of the monkey calculated daily using a simple delayed reach task to yield an estimate of the DT in a given trial (vertical red dashed line). Knowing the DT, we can estimate the success probability at which the monkey committed to his choice in a given trial (horizontal red dashed line). C, Example success probability profiles of "easy" (blue), "ambiguous" (green), or "misleading" (red) trials, classified a posteriori according to the following criteria: A trial is classified as "easy" if success probability (SP) exceeds 0.6 after tw0 token jumps and 0.75 after five token jumps. A trial is ambiguous if the SP is 0.5 after two jumps, between 0.4 and 0.65 after three token jumps, and then between 0.55 and 0.66 after five and seven jumps. A trial is misleading if SP is $<0.4$ after three token jumps. $\boldsymbol{D}$, Reconstructed images of the brain surface from anatomical MRI scans. Large circles represent the location of the recording chambers. Colored circles represent the location of the recording sites for cells recorded in the two blocks of trials (211 cells in PMd, 175 cells in M1), classified as PMd (blue) or M1 (red) based on anatomical location. A, Anterior; P, posterior; M, medial; L, lateral.

calculated this quantity (with respect to the target ultimately chosen by the monkey) for each token jump (Fig. $1 B$ ). For example, if at a particular moment in time the right target contains $N_{R}$ tokens, the left contains $N_{L}$ tokens, and $N_{C}$ tokens remain in the center, then the probability that the target on the right will ultimately be the correct one (i.e., the success probability of guessing right) is as follows:

$$
p\left(R \mid N_{R}, N_{L}, N_{C}\right)=\frac{N_{C} !}{2^{N_{C}}} \sum_{k=0}^{\min \left(N_{C,} 7^{-N_{L}}\right)} \frac{1}{k !\left(N_{C}-k\right) !}
$$

Although each token jump and each trial were completely random, we could classify a posteriori some specific classes of trials embedded in the fully random sequence (e.g., "easy," "ambiguous," or “misleading” trials, Fig. 1C). RT was calculated as the time of movement onset (based on kinematics) and the time of the first token jump. Decision time (DT) was estimated by subtracting from the RT the monkey's mean RT from the delayed reach task performed on the same day. We could then compute for each trial the duration of a decision as well as its success probability at the time of the decision (Fig. 1B). Wilcoxon-Mann-Whitney (WMW) tests were used to compare RT, DD, or success probability distributions.

Calculation of the monkey's accuracy criterion at DT relies on the available sensory evidence at that time. Because it is unlikely that the monkeys could compute Equation 1, we calculated a simple "first-order" 
approximation of sensory evidence as the sum of log-likelihood ratios (SumLogLR) of individual token movements as follows:

$$
\operatorname{SumLogLR}(n)=\sum_{k=1}^{n} \log \frac{p\left(e_{k} \mid S\right)}{p\left(e_{k} \mid U\right)}
$$

where $p\left(e_{k} \mid S\right)$ is the likelihood of a token event $e_{k}$ (a token jumping into either the selected or unselected target) during trials in which the selected target $S$ is correct, and $p\left(e_{k} \mid U\right)$ is its likelihood during trials in which the unselected target $U$ is correct. The SumLogLR is proportional to the difference in the number of tokens that have moved in each direction before the moment of decision (for more details on this analysis, see Cisek et al., 2009). To characterize the decision policy of a given monkey in a given block of trials, we binned trials as a function of the total number of tokens that moved before the decision, and calculated the average SumLogLR for each bin.

Above, we defined "sensory evidence" as the information, pertinent to the correct choice, which is continuously present within the stimulus (i.e., the number of tokens in each target). This is analogous to the motion signal continuously present within the stimulus of random-dot motion discriminations tasks (Britten et al., 1992), and to the total probability indicated by the visible symbols in the task of Yang and Shadlen (2007). In contrast, individual token jumps are novel pieces of information that change the sensory evidence, analogous to the appearance of a new symbol in the task of Yang and Shadlen (2007) or to each new auditory click in the click-counting task of Brunton et al. (2013). In previous publications, we suggested that the brain only integrates that novel information (Cisek et al., 2009; Thura et al., 2012). For example, we have recently shown that, during random-dot motion discrimination, the motion signal is not integrated over time, as usually assumed (Carland et al., 2015), and that what is instead integrated are the changes in the motion signal (Thura et al., 2012).

All arm and eye movement data were analyzed off-line using MATLAB (The MathWorks). Reaching characteristics were assessed using monkeys' movement kinematics. Horizontal and vertical position data were first differentiated to obtain a velocity profile and then filtered using a sixth-order low-pass filter with a frequency cutoff of $15 \mathrm{~Hz}$. Onset and offset of movements were determined using a $3 \mathrm{~cm} / \mathrm{s}$ velocity threshold. Peak velocity was determined as the maximum value between these two events.

Neural data analysis. All neurophysiological data reported here were acquired from correct or error trials in which the monkeys completed the tokens task by choosing one of the two targets. To be included in the analyses, neurons had to be recorded during at least 100 trials in each of the slow and the fast blocks (in one or more repetitions of each block).

Neurons were selected according to their anatomical location and physiological properties (Fig. 1D). Among all cells recorded in PMd and M1, we first focus on those showing a significant spatial preference for one of the targets during the deliberation process (i.e., between the first token jump and our estimate of DT). In a recent study (Thura and Cisek, 2014), we showed that these cells reflect the deliberation process by tracking the evolution of sensory evidence and then signal the commitment to the choice $\sim 280 \mathrm{~ms}$ before movement execution. For each of these cells, we calculated the mean activity for each target choice during the $200 \mathrm{~ms}$ preceding DT in the tokens task and assessed the significance using a receiver-operating characteristic (ROC) (Green and Swets, 1966; Shadlen et al., 1996) analysis with a criterion of 0.65 .

The effects of block on these decision-related cells ( $\mathrm{ROC}>0.65)$ were assessed via comparisons of averaged neural activity in various epochs of the task (see Results). In each individual cell, robustness of the effect of block was assessed with a WMW statistical test (which compares neural activity distributions). For a subset of cells, we investigated the relationship between sensory evidence (calculated as SumLogLR) and neural activity as a function of DD (in bins of $200 \mathrm{~ms}$ corresponding to the token jumps) in the two blocks of trials. Spearman's rank test was used to assess significance of the relationship between SumLogLR and neural activity, and analysis of covariance (ANCOVA) was used to assess the interaction between blocks and DD on neural activity. From this analysis, we focused on the evolution of the averaged activity in PMd and M1 for the condi- tion when SumLogLR $=0$ (equal evidence for each target) as a function of time (after each token jump, every $200 \mathrm{~ms}$ bin) in either the slow or the fast blocks. The average activity was calculated by averaging means across cells for bins in which data were available (even-numbered jumps). For odd-numbered jumps, the mean activity at zero evidence was estimated for each cell via interpolation of data surrounding SumLogLR $=0$ and the population average activity was computed by averaging these means. To assess the robustness of the effect of block on the population activity at $\operatorname{SumLogLR}=0$, we performed a bootstrap test for bins in which data at this time were available (even-numbered jumps). The bootstrap procedure consisted of resampling the firing rate data of each cell 10,000 times in each bin and in the two block conditions to produce distributions of means and a distribution of the difference in the means. If zero lies outside the $2.5 \%-97.5 \%$ percentiles of the distribution of resampled differences, then the effect is considered significant at $p<0.05$.

Next, we compared neural activity in the two blocks of trials in all cells showing a significant correlation (Pearson's linear correlation coefficient) between (1) firing rate and DD and (2) firing rate and peak velocity of the reaching movement (see Results). Average neuronal activity correlated with DD or movement peak velocity was computed for each block and compared in two groups of trials defined by the DDs. As above, significance of the effects was assessed by a bootstrap procedure.

Instantaneous firing rate was assessed via a partial interspike interval method. When analyzing data with respect to the start of the trial (first token jump), we always exclude all spikes occurring after our estimate of DT (i.e., any activity associated with movement initiation and/or execution). This is important to prevent averaging artifacts due to the very wide range of DDs in the tokens task.

The significance level of all statistical tests was set at 0.05 .

\section{Results}

\section{Effect of block on monkeys' behavior}

The primary goal of the present paper is to assess the neural mechanisms of the SAT adjustments induced by the timing parameters of the task. After extensive training, both monkeys adapted their behavior as a function of the block ("slow" or "fast"). In a recent report, we described their behavior in detail both during and after they adapted their decision and movement policies (Thura et al., 2014). Here, we focus our work on data collected after each monkey showed strong and consistent behavior that differed in the two block conditions. Typically, this adaptation consists of a significant reduction of DDs and a decrease of success probabilities in fast blocks compared with slow blocks. Figure $2 A$ illustrates an example session of Monkey Z's behavior as a function of the block condition.

Across all trials during which neurons described in the present paper were recorded, Monkey $\mathrm{S}$ and Monkey $\mathrm{Z}$ made decisions on average 491 and $534 \mathrm{~ms}$ faster in the fast blocks compared with the slow blocks, respectively (WMW test, $p<0.001$ for both monkeys). This time savings was accompanied by a reduction of the overall performance in the fast blocks compared with slow blocks, for both monkeys $(73.9 \%$ vs $79.9 \%$ of correct choices for Monkey S; 70.7\% vs $76.9 \%$ of correct choices for Monkey Z).

This trade-off was very consistent between sessions. Figure $2 B$ shows, for both monkeys and for all sessions during which neurons are analyzed below ( 49 sessions in Monkey S, 81 sessions in Monkey Z), the mean RTs in the fast versus the slow block condition. Both animals made decisions significantly earlier in fast blocks compared with slow blocks in all recording sessions (WMW test, $p<0.05$ ), except for 2 of 49 and 3 of 81 sessions in Monkey S and Monkey Z, respectively. As expected, in most of these sessions, success probability as well as performance were lower during fast blocks compared with the slow blocks (Fig. $2 C, D)$. Thus, monkeys traded speed for accuracy between the blocks. Did they also accomplish this adjustment within blocks? 
A

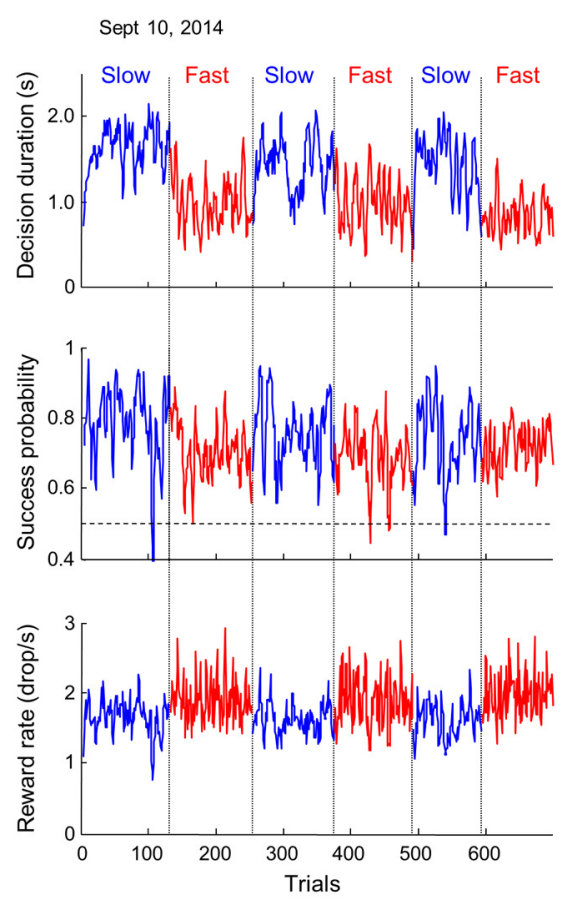

B

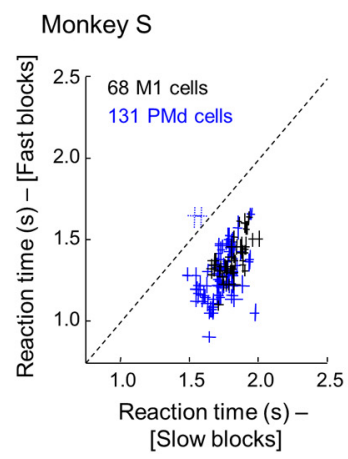

Monkey Z

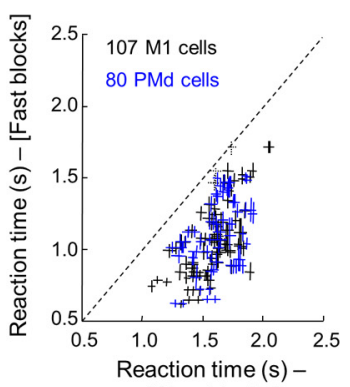

[Slow blocks]
C
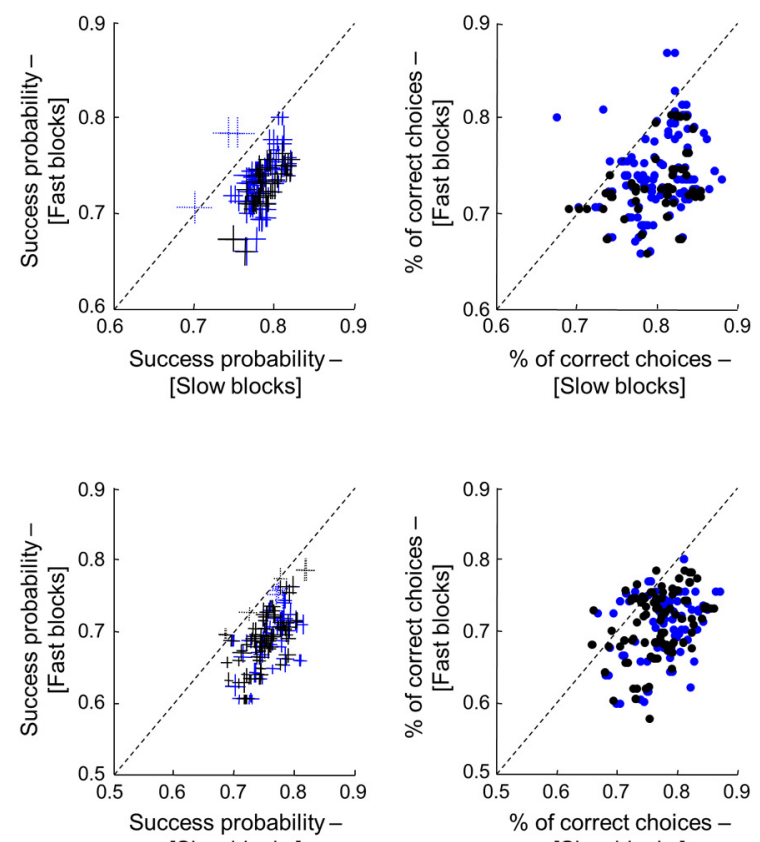

[Slow blocks]

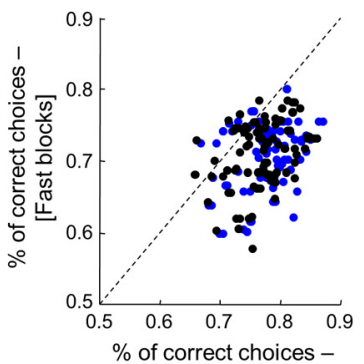

[Slow blocks]

Figure 2. A, Example session performed by Monkey Z, showing the DDs, success probability at decision (SPD), and reward rate (RR) (for details about reward rate calculation, see Thura et al., 2014) for consecutive trials performed in slow (blue) and fast blocks (red). $\boldsymbol{B}$, For each of the recorded cells in either PMd (blue) or M1 (black), comparison of the mean ( \pm SE) RTs of the trials performed in the slow blocks (abscissa) or fast blocks (ordinate) in Monkey $S$ (top) and Monkey Z (bottom). Dotted crosses indicate session during which RTs were not significantly different in the two blocks. $\boldsymbol{C}$, Same as $\boldsymbol{B}$ for the mean ( $\pm \mathrm{SE}$ ) success probability at DT. $\boldsymbol{D}$, Same as $\boldsymbol{B}$ for the performance, calculated as the percentage of correct choices in a given session.

We estimated the "accuracy criterion" for committing to a choice in a given block by computing, for each monkey and each block type, the available sensory evidence for the chosen target at the time of the decision as a function of DD (see Materials and Methods) (Cisek et al., 2009). Figure 3A shows that, for both animals and in both blocks, the average level of sensory evidence at the time of commitment first increases and then (after $\sim 900$ $\mathrm{ms}$ ) decreases as a function of DD. Although this pattern appears complex, it can be easily explained with a purely linear urgency signal with intertrial noise: Early decisions $(<900 \mathrm{~ms}, 23 \%$ in Monkey S, 38\% in Monkey Z) occur only when noise happens to be high, leading to an early guess even when evidence is low; hence, the early part of the curve is low and rises with time. Later decisions are more common (77\% and 62\% in Monkey S and Monkey Z, respectively) and the mean evidence level provides an accurate estimate of the animal's "accuracy criterion" at that time. Thura et al. (2014) simulate these data (Fig. 3A, dashed lines) using a UGM with the purely linear urgency signals shown here in Figure $3 B$.

An accuracy criterion that drops over time implies that, if sensory information is strong, the monkeys usually decide quickly. If information is ambiguous, they wait to see if it improves; and finally, if too much time has passed, they make a guess. We found that, for both monkeys, the estimated urgency signal is significantly higher during fast blocks than slow blocks. This suggests that, as expected, the monkeys are more willing to guess in the fast blocks and wait longer to decide in the slow blocks.

As stated in Materials and Methods, monkeys usually started each session with the slow block of trials. It is thus conceivable that the effects described above (faster RTs and lower accuracy in fast blocks) are related to fatigue or to a decreasing attention/ motivation over the course of the session. To control for these potential confounds, we tested animals in sessions in which two blocks of slow trials surrounded a fast period. The results illustrated in Figure $3 C$ show that Monkey $S$ recovers almost entirely a slow-type pattern in the second of two slow blocks (following the fast block), ruling out the possibility that effects of blocks are due to factors such as motivation or satiety. Monkey Z shows the same trend but without a complete recovery. In the second slow block (cyan line), he was usually a little more willing to tolerate less sensory evidence and decide faster than in the first slow block (blue line). Nevertheless, his behavior was still significantly different and more conservative than in the preceding fast block (red line).

Overall, these behavioral data show that the context of the task (fast vs slow block) strongly affects monkeys' behavior and that they voluntarily establish (within a given block) and adjust (between blocks) their SAT. For both monkeys, the urgency signals derived from their choice behavior grow as a function of time in both blocks, but the initial level of urgency is higher in the fast blocks and then converges to the level of the slow blocks late in the trials. This makes good sense because the difference in the time savings afforded by each block is smaller as the number of tokens remaining in the center diminishes with time.

Can we find a neural signature of these patterns of urgency in the activity of PMd and M1 cells during decision-making and movement execution? In particular, the UGM predicts that, for a given level of sensory evidence, neural activity should be higher in the fast than the slow blocks at the start of each trial, then gradually rise over time but at a slower rate in fast blocks, eventually converging to the same level in both blocks (Fig. 3B). Furthermore, assuming that commitment is determined through a competition within PMd and M1 (Cisek, 2007; Thura and Cisek, 
A

B

\section{Monkey S}

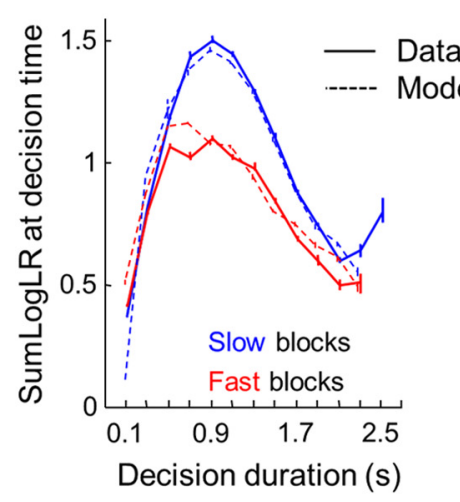

Monkey Z

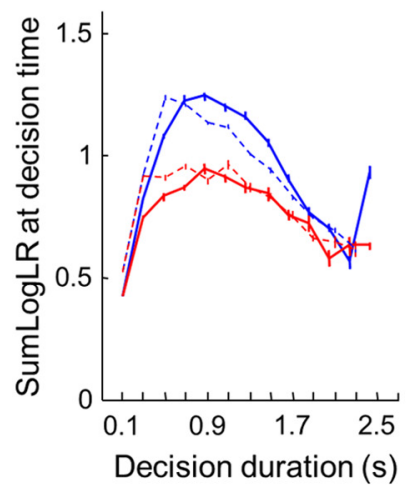

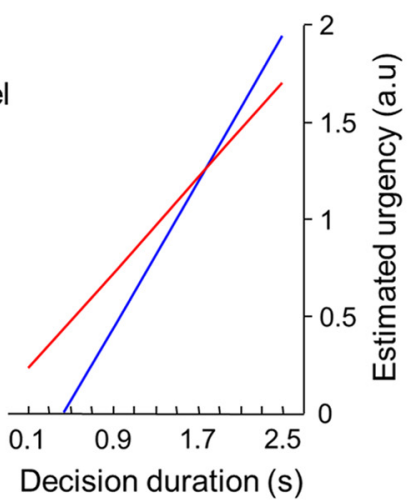

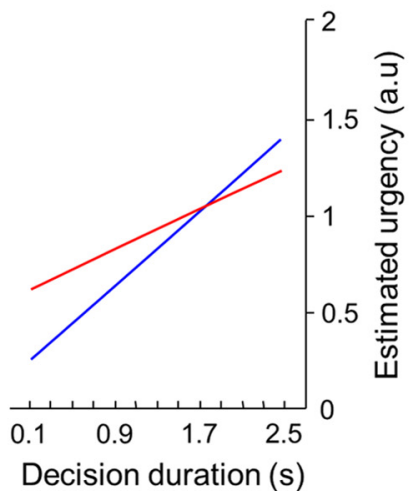

C

First block : slow \#1

Second block: fast \#1

Third block : slow \#2
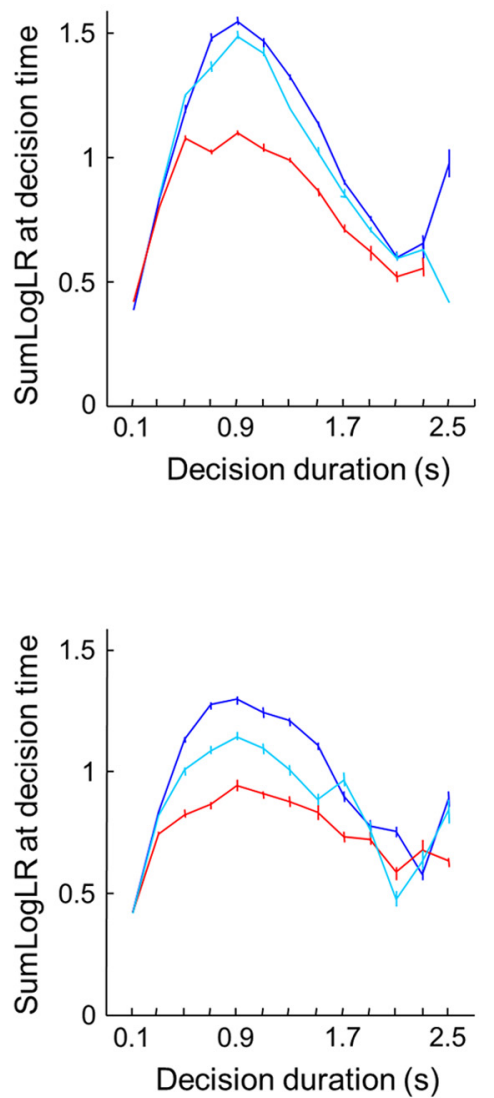

Figure 3. $A$, Mean ( \pm SE) of the SumLogLR (see Eq. 2) at DT, used to estimate the accuracy criterion as a function of DD (in $200 \mathrm{~ms}$ bins) during slow (blue) and fast (red) blocks. Top, Monkey S. Bottom, Monkey Z. B, For each monkey, the estimated urgency functions in the slow (blue) or the fast (red) blocks derived from the UGM. C, Same as $\boldsymbol{A}$, but here slow block trials are separated depending on whether the slow block was the first of the session (slow block 1, blue) or the third (slow block 2, light blue), having followed an intervening fast block (red).

2014), neural activity at the time of commitment should be the same across blocks as well as across trials.

\section{Decision-related neural activity in PMd and M1}

Here, we analyze the activity of 211 PMd (131 in Monkey S) and 175 M1 (68 in Monkey S) cells that were recorded in at least 100 trials during each of the slow and fast blocks (Table 1). Because the results of all analyses were qualitatively the same regardless of which arm the monkey used to perform the task, we combined all results across neurons in both hemispheres in each area. Most of the neurons were recorded while monkeys performed a slow block followed by a fast block ( 371 of 386 cells). To control for potential confounds related to evolving signals or motivation, 228 of these cells were tested in at least one additional block of slow trials following the fast block; and of these, 55 cells were tested in two or more repetitions of both slow and fast blocks.

First, we compared neuronal activity between slow and fast blocks in PMd and M1 cells that discriminate the chosen target during the deliberation process. These cells were the subject of a recent paper showing that they are strongly involved in decision deliberation as well as choice commitment (Thura and Cisek, 2014). Here, we study these "decision-related" cells (PMd, $n=$ 61; M1, $n=78$ ) tested in enough trials in the two blocks, both before (baseline) and during the deliberation process. First, we found that average activity patterns during easy, ambiguous, and misleading trials (Fig. 1C) performed in the slow blocks replicate our previous observation: these cells track the evolution of sensory evidence guiding the choice and reach a peak of activity before the onset of movements ( $\sim 280 \mathrm{~ms}$ in PMd, $\sim 140 \mathrm{~ms}$ in M1) expressing that choice (Fig. 4). Next, we found that, overall, a large majority of these PMd (44 of 61, 72\%) and M1 (58 of 78, $74 \%$ ) cells were significantly modulated by the block condition before and/or during the deliberation process (significant WMW test on at least one of the epochs described below). In the majority of cases ( 30 of 44, 68\% in PMd; 47 of 58, 81\% in M1), activity was higher in the fast blocks compared with the slow blocks. Examples of such block-sensitive cells in both PMd and M1 are illustrated in Figure 5. Figure 5A, $B$ shows an example $\mathrm{M} 1$ cell that was tested in two repetitions of slow and fast blocks. Baseline activity is unaffected by these changes, but the cell's response to token jumps is strongly influenced by the block condition, regardless of the ultimate direction chosen by the monkey. It exhibits a strong burst in the fast blocks, whereas it only slowly rises in the slow blocks. The PMd cell depicted in Figure $5 C, D$ shows a similar effect. Here, activity is modulated by the block condition both during the baseline and the deliberation period. Furthermore, unlike the M1 cell shown above, in the second block of slow trials, the activity of this PMd cell does not come back to the same level observed during the first slow block, especially for the baseline period. This may reflect the tendency of monkeys to become 
Table 1. Numbers of recorded neurons as a function of their location (PMd or M1) ${ }^{a}$

\begin{tabular}{|c|c|c|}
\hline & PMd, no. (\%) & $\overline{M 1, \text { no. }(\%)}$ \\
\hline Total recorded cells & 273 & 207 \\
\hline Total recorded cells in both slow and fast blocks & 211 & 175 \\
\hline MonkeyS & 131 & 68 \\
\hline Monkey Z & 80 & 107 \\
\hline $\begin{array}{l}\text { Cells significantly modulated by sensory } \\
\text { evidence ("decision" cells) }\end{array}$ & $61 / 211$ & $78 / 175$ \\
\hline Cells significantly modulated by DD & $63 / 211$ & $53 / 175$ \\
\hline Positively correlated & $31 / 63$ & $24 / 53$ \\
\hline Decision cells significantly correlated with DD & $18 / 61$ & $25 / 78$ \\
\hline $\begin{array}{l}\text { Cells significantly modulated by movement } \\
\text { speed }\end{array}$ & $19 / 211$ & $18 / 175$ \\
\hline MonkeyS & 10 & 9 \\
\hline Monkey Z & 9 & 9 \\
\hline Decision cells tested during the baseline period & $61 / 61$ & $78 / 78$ \\
\hline $\begin{array}{l}\text { Cells modulated by the blocks during the } \\
\text { baseline period }\end{array}$ & $34 / 61(56)$ & $46 / 78(59)$ \\
\hline $\begin{array}{l}\text { Higher activity in the fast blocks during the } \\
\text { baseline period }\end{array}$ & $22 / 34(65)$ & $35 / 46(76)$ \\
\hline $\begin{array}{l}\text { Decision cells with at least } 5 \text { easy trials in } \\
\text { PT: DT }>400\end{array}$ & $40 / 61$ & $50 / 78$ \\
\hline Modulation by the blocks & $6 / 40(15)$ & $9 / 50(18)$ \\
\hline Higher activity in the fast blocks & $6 / 6(100)$ & $8 / 9(89)$ \\
\hline $\begin{array}{l}\text { Decision cells with at least } 5 \text { ambiguous } \\
\text { trials (PT or OT): DT }>600\end{array}$ & $47 / 61$ & $63 / 78$ \\
\hline Modulation by the blocks & $10 / 47(21)$ & $9 / 63(14)$ \\
\hline Higher activity in the fast blocks & $8 / 10(80)$ & $7 / 9(78)$ \\
\hline $\begin{array}{l}\text { Decision cells with at least } 5 \text { misleading trials in } \\
\text { OT: DT }>900\end{array}$ & $52 / 61$ & $67 / 78$ \\
\hline Modulation by the blocks & $13 / 52(25)$ & $19 / 67(28)$ \\
\hline Higher activity in the fast blocks & $9 / 13(69)$ & $16 / 19(84)$ \\
\hline $\begin{array}{l}\text { Decision cells modulated by the blocks in at } \\
\text { least one epoch }\end{array}$ & $44 / 61(72)$ & $58 / 78(74)$ \\
\hline Higher activity in the fast blocks & $30 / 44(68)$ & $47 / 58(81)$ \\
\hline $\begin{array}{l}\text { Decision cells modulated around the peak of } \\
\text { activity }\end{array}$ & $33 / 61(54)$ & $32 / 78(41)$ \\
\hline Higher activity in the fast blocks & $20 / 33(61)$ & $19 / 32(59)$ \\
\hline
\end{tabular}

${ }^{a}$ For each area, neuron counts are further classified based on their modulation by sensory evidence, DD, or movement speed. Finally, the numbers (and percentages) of units modulated by the block condition (slow vs fast) during the different epochs of the trial are summarized.

hastier over the course of a daily session, as depicted above in Figure $3 C$, perhaps reflecting gradual devaluation of reward.

To quantify the effect of block at the population level, we first examined activity during a baseline period defined as the $200 \mathrm{~ms}$ preceding the first token jump. For this analysis, both trials in which monkeys chose the cell's preferred (PT) or nonpreferred target (OT) were included, as no effect of evidence could affect activity at that time. The average response of the 61 "decisionrelated" PMd cells in both blocks is shown in Figure 6A (left). This plot shows that during fast blocks, activity is slightly increased compared with slow blocks. A cell-by-cell analysis revealed that approximately half of the cells (34 of 61, 56\%) were significantly modulated by the block condition. Among these, we found that $22(65 \%)$ had a significantly higher baseline activity in the fast blocks compared with the slow blocks (Fig. $6 B$, left). In M1, baseline activity was also modulated as a function of the block condition in more than half of the cells (46 of 78,59\%), with activity usually stronger in fast blocks (35 of 46, 76\%; Fig. $6 D$, left), resulting in an overall slight increase of baseline activity during fast blocks (as shown on the M1 population average response in the left panel of Fig. 6C).

Next, we assessed the effect of block on neural activity during the deliberation process (discarding all activity after DT to pre- vent contamination by preparatory and execution processes). As trials were highly variable in terms of sensory evidence provided to the animals, we classify them according to their success probability profiles and focused our analyses on easy, ambiguous, and misleading trials (see Materials and Methods). In a previous report, we showed that both PMd and M1 cells reflected the evolution of the sensory evidence used by the monkey to make a choice. Indeed, regardless of the block, Figure $6 A, C$ (three right panels) clearly shows that cells quickly discriminate the cell's PT in easy trials, discrimination takes longer in ambiguous trials, and in misleading trials, a switch of activity is observed, reflecting the inversion of success probability in such trials.

What happens when we compare this evidence-related activity between the slow and fast blocks? On average, population activity was strongly amplified during fast blocks compared with slow blocks, especially in easy and misleading trials, as illustrated in Figure $6 A, C$ for both populations. In ambiguous trials, modulations appeared to be weaker at the population level, especially in PMd (Fig. 6A, third panel from left). Nevertheless, these results suggest that these cells are influenced by both the sensory evidence and by a block-dependent signal.

Next, we conducted a cell-by-cell analysis of all neurons recorded in at least 5 trials of a given trial type in each of the two blocks. To choose the time epochs for this analysis, we first visually inspected the time period during which population responses reflected trial types, and then made sure that these periods preceded decision commitment (Fig. 6A,C). Thus, for easy trials, we compared activity during a $200 \mathrm{~ms}$ time window shortly following the deliberation onset (from 200 to $400 \mathrm{~ms}$ after the first token jump); for ambiguous trials, we focused on the ambiguous epoch (from 400 to $600 \mathrm{~ms}$ after the first token jump); and for misleading trials, we focused on the epoch when neural activity tended to favor the target opposite to each cell's PT (from 700 to $900 \mathrm{~ms}$ after the first token jump). Given these constraints, only PTrelated easy trials with DDs $>400 \mathrm{~ms}$ were considered (PMd: $n=$ 40; $\mathrm{M} 1=50$ ); for ambiguous trials, both PT and OT-related trials with DDs > $600 \mathrm{~ms}$ were considered (PMd: $n=47$; $1=63$ ); and for misleading trials, only OT-related trials with DDs $>900$ ms were considered (PMd: $n=52 ; \mathrm{M} 1=67$ ).

Results depicted in Figure $6 B, D$ (three right panels) show that a subset of PMd cells (6 of 40,15\%) were significantly modulated by the block condition shortly after deliberation onset during easy trials. Among them, all had significantly higher activity in the fast blocks compared with the slow blocks. In M1, activity during easy trials was also modulated as a function of the block condition in a subset of cells ( 9 of $50,18 \%$ ), with activity also almost always stronger during fast blocks (8 of 9, 89\%). In ambiguous trials, 10 of 47 of the PMd cells and 9 of 63 of the M1 cells were significantly modulated by the block, usually ( 8 of 10 in PMd; 7 of 9 in M1) showing an increase of activity in the fast blocks compared with the slow blocks. Finally, during misleading trials, one-fourth of PMd cells (13 of 52) were significantly modulated by the block condition during the tested $200 \mathrm{~ms}$ epoch. Among them, we found that 9 of 13 (69\%) had significantly higher activity in the fast blocks compared with the slow blocks. In M1, 19 cells were modulated by the block condition in misleading trials $(28 \%)$, with activity usually stronger in fast blocks (16 of $19,84 \%)$.

Because neural activity related to cells' PT peaks close to commitment time (Fig. 4), the higher neural activity usually observed in the fast blocks could reflect the rise of this activity to the peak in trials in which choice commitments occurred close to the analysis window. Although this cannot be responsible for the large effect 

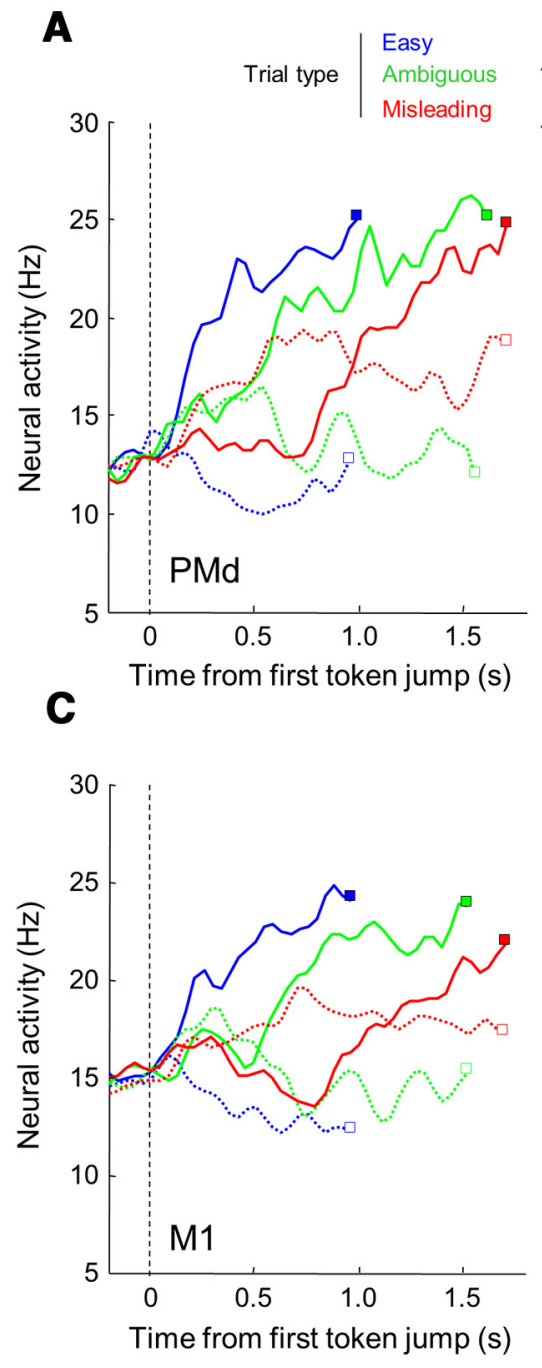

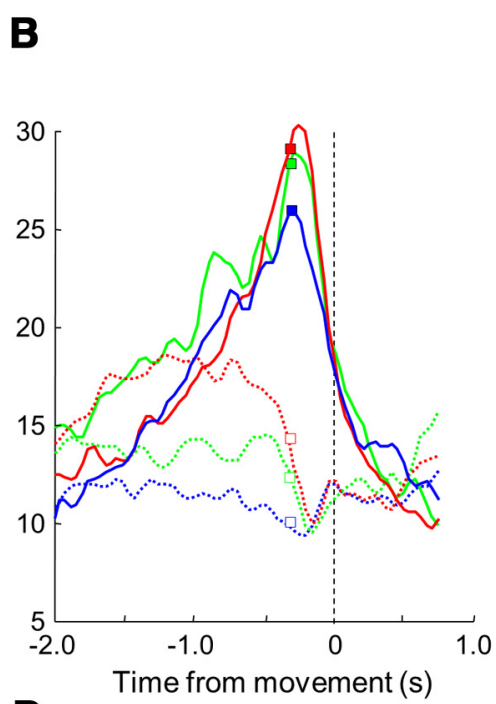

D

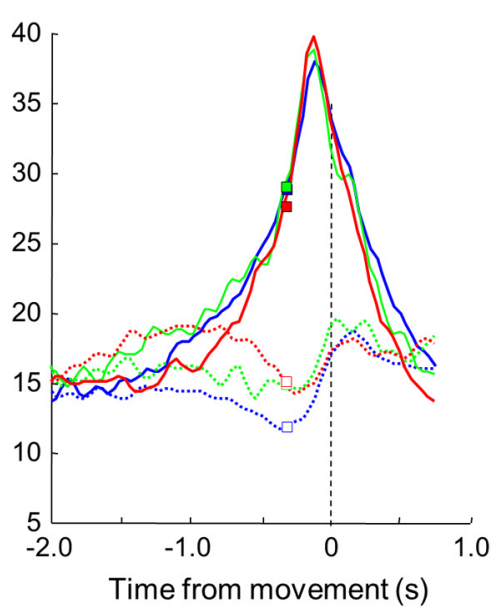

Figure 4. A, Average activity of spatially tuned PMd neurons during easy (blue, $n=61$ ), ambiguous (green, $n=40$ ), and misleading (red, $n=61$ ) trials in which the monkey correctly chose the recorded cell's preferred target (solid lines) or the opposite target (dotted lines) in the slow blocks. Activity is aligned on the first token jump and truncated 280 ms before movement onset (squares) to avoid averaging artifacts. To be included in the analysis, cells had to be recorded during at least 5 trials in each trial type and direction. $\boldsymbol{B}$, Average activity of the same PMd cells aligned on movement onset. Squares represent our estimate of the monkey's commitment time. C, Same as $\boldsymbol{A}$ for a population of spatially tuned M1 cells ( $n=78$ in easy trials, $n=60$ in ambiguous trials, and $n=78$ in misleading trials). $\boldsymbol{D}$, Average activity of the spatially tuned M1 cells aligned on movement onset.

In Figure 7A, $C$ (for PMd and M1 cells, respectively), we compare activity between a slow block that preceded a fast block. As noted above, we observe an amplification of activity in the fast block. In Figure $7 B, D$, the comparison is between a second slow block that followed a fast block. In this second block of slow trials, the activity is reduced in comparison with the fast block, consistent with the proposal that the amplitude changes are primarily attributable to SAT adjustments.

The results described above suggest that the activity of a large population of PMd and M1 decision-related cells is amplified during fast blocks, when animals favor decision speed over accuracy. But is activity during the early part of the deliberation process a good predictor of monkeys' subsequent RTs within blocks? To answer this question, we performed a trial-by-trial correlation between DD and activity related to cells' PT from 200 to 500 ms following the first token for each PMd and M1 decision-related cell in separate slow and fast blocks. We found that, for a subpopulation of cells (PMd: $18 \%$ in slow block, $23 \%$ in fast block; M1: $28 \%$ in slow block, $19 \%$ in fast block), early deliberation-related activity is significantly correlated with DD. As expected, correlations are usually negative, meaning that the stronger the firing rate, the shorter the decision. Although these percentages are not negligible and may implicate trial-to-trial variability in urgency as a factor influencing RTs, it is probably only one of many other factors (e.g., sensory evidence, fluctuations in attention) that determine the RT on any particular trial.

Using the UGM, we can estimate the shape of the urgency signal in the two blocks on the basis of each animal's be-

of block that we observed on baseline activity in PMd and M1, it could potentially confound activity during deliberation, especially in easy trials. To control for this, we performed additional comparisons where we restricted our analyses to trials in which RT was much longer than the analysis windows (RTs $>1000 \mathrm{~ms}$ and RTs $>1200 \mathrm{~ms}$ ). In both cases, we found similar results (data not shown), although the effects at the level of many individual cells did not meet significance because of the large number of discarded trials.

Another important control consists of assessing the effects of recording stability and/or monkeys' evolving motivation/ fatigue during the time course of a recording session. As neurons were usually recorded in a slow block of trials first, the observed amplification of signals in fast blocks could reflect factors other than SAT adjustment. To examine this issue, most of the decision neurons in PMd and M1 were tested in a second block of slow trials following the fast block. If modulations of activity are related to SAT adjustment, then activity should recover a slow-type pattern after the fast condition. Figure 7 clearly shows this result. havior (Fig. 3B) (for details, see Thura et al., 2014). Is it possible to observe a neural signature of these shapes from the blockmodulated cells? To answer this question, we looked at the correlation between cell activity and sensory evidence (calculated as the SumLogLR) as a function of time in each of the two blocks (Fig. 8). As previously reported (Thura and Cisek, 2014), both PMd and M1 cells are positively correlated with sensory evidence, from the beginning of the deliberation until the time of commitment. Here, we focus on cells that were significantly modulated by the block condition, with firing rate higher in the fast blocks compared with the slow blocks, during at least one of the tested periods (baseline period in all trials, $200-400 \mathrm{~ms}$ in easy trials, $400-600 \mathrm{~ms}$ in ambiguous trials, $700-900 \mathrm{~ms}$ in misleading trials; PMd, $n=30$; M1, $n=47$ ). The comparison of the correlation in the two conditions shows, as expected, that both PMd and M1 activity is usually stronger in the fast blocks than in the slow blocks (Fig. 8A,C). More interestingly, if we examine the neuronal activity at the zero evidence point, and then plot it as a function of time in the two conditions (Fig. $8 B, D$ ), we observe that, 
A

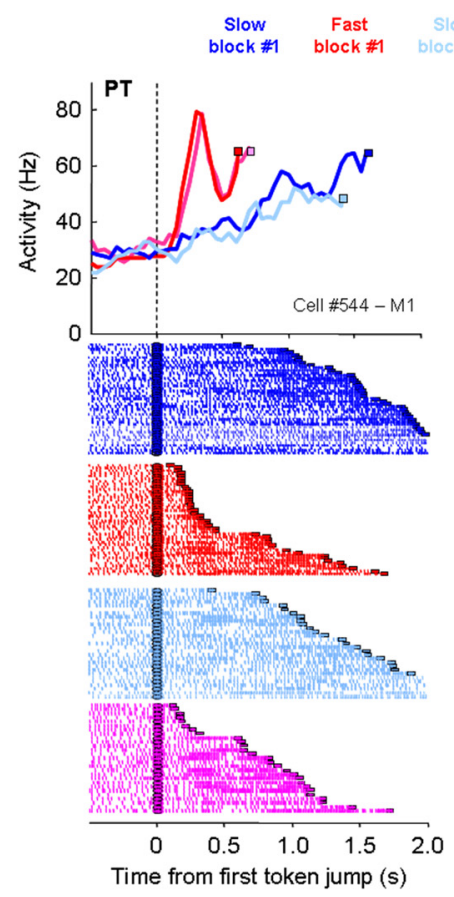

B

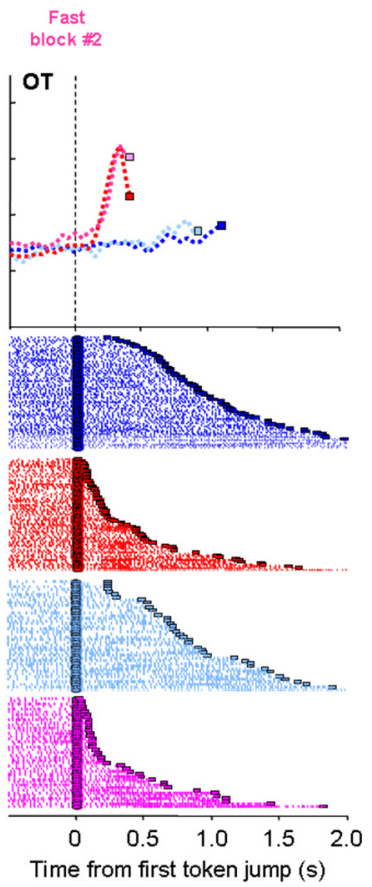

C

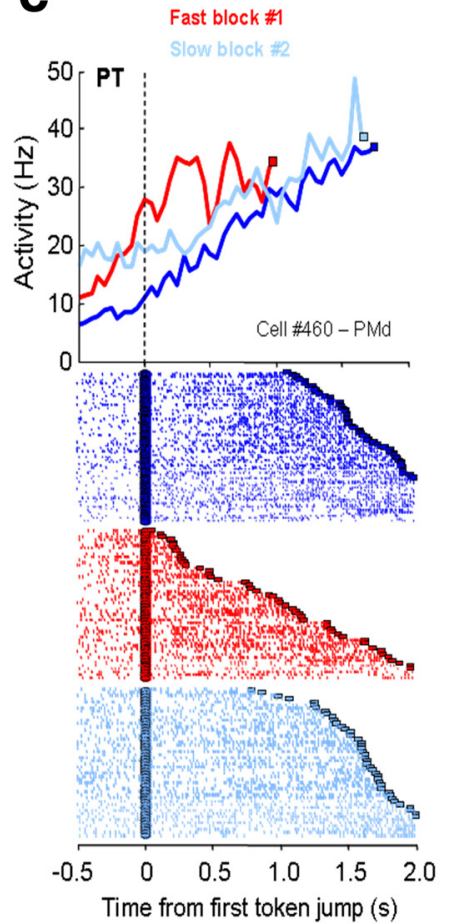

D

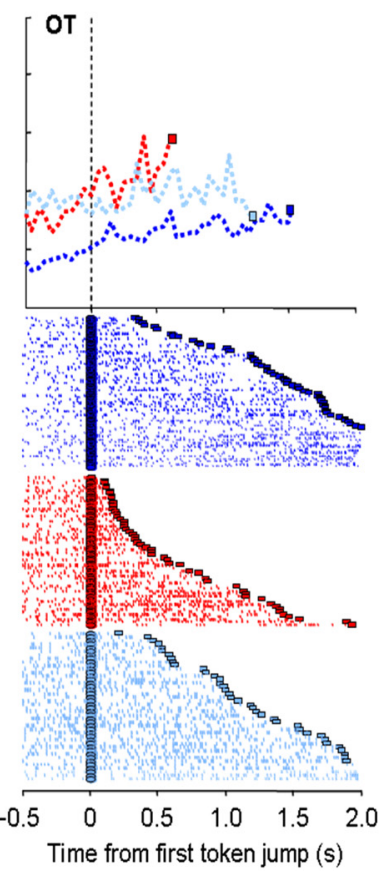

Figure 5. A, Response of an M1 neuron tuned before commitment when the monkey chose the cell's PT. Top, Average activity across trials, grouped as a function of the block, aligned on the first token jump and truncated $280 \mathrm{~ms}$ before movement onset (squares). The neuron was first tested in one slow block (blue), then in a fast block (red), in a second slow block (light blue), and finally in a second fast block of trials (magenta). All trial types in both the fast and the slow blocks are included, and rasters (bottom panels) illustrate the neural activity (from $500 \mathrm{~ms}$ before the first token jump to $280 \mathrm{~ms}$ before movement onset) of all these individual trials in each of the tested blocks, sorted by DD. $\boldsymbol{B}$, Same as $\boldsymbol{A}$ when the monkey chose the 0T. C, Response of a PMd neuron tuned before commitment (compare with $\boldsymbol{D}$ ) when the monkey chose the cell's PT. Top, Average activity across trials, grouped as a function of the block, aligned on the first token jump and truncated $280 \mathrm{~ms}$ before movement onset (squares) to avoid averaging artifacts. The neuron was first tested in a slow block (blue), then in a fast block (red), and finally in a second slow block (light blue). All trial types in both the fast and the slow blocks are included, and rasters (bottom panels) illustrate the neural activity of all these individual trials in each of the tested blocks, sorted by DD. D, Same as C when the monkey chose the $0 \mathrm{~T}$.

for PMd cells, activity is growing regardless of the block (ANCOVA, time, $F=23.9, p<0.0001$ ) and is higher in the fast blocks (ANCOVA, block, $F=7.41, p=0.0067$ ), especially early in the trial. Nevertheless, the interaction between time and block is not statistically significant (ANCOVA, time $\times$ block, $F=0.77$, $p=0.38)$. This is reminiscent of the urgency shapes we derived from monkeys' behavior. In M1, we observe a similar effect (ANCOVA, block, $F=10.6, p=0.0012$; time $\times$ block, $F=0.87$, $p=0.35)$, although the activity does not grow over time as much as it does in PMd (ANCOVA, time, $F=9.7, p=0.0019$ ).

We performed several control analyses to assess the robustness of these important results. First, we normalized activity of each cell (based on the mean firing rate computed across blocks in a $60 \mathrm{~ms}$ window around commitment time, i.e., $310-250 \mathrm{~ms}$ before movement onset) before averaging across populations. The results (data not shown) were qualitatively the same as without normalization. Next, as for the analysis depicted in Figure 6, we controlled for a potential influence of commitment-related activity on the observed modulations. We performed the same analysis using only trials with RTs between 1000 and $1500 \mathrm{~ms}$ in both blocks, to see if activity early in the trials (i.e., far from decision commitment) was still modulated by the SAT condition. We found that, even in this highly constrained condition, activity at zero evidence still rises with time and is still higher in the fast than the slow blocks (ANCOVA shows all effects described above significant at $p<0.05$ ). Finally, to verify that both monkeys generalized SAT performance across limbs, we did that analysis again by grouping cells according to the hemisphere recorded. The results show that modulation by the time- and block-related signal is valid for both PMd and M1 populations regardless of the hemisphere sampled (especially in Monkey $\mathrm{Z}$ in which many modulated cells were collected in each hemisphere). Together, these results suggest that both PMd and M1 cells are influenced by the evolving evidence and by a context-dependent, timerelated signal, in agreement with the UGM (Cisek et al., 2009; Thura et al., 2014).

\section{Decision-related neural activity in PMd and M1 at commitment time}

In a previous article (Thura and Cisek, 2014), we demonstrated that, when the activity of decision-related cells is aligned on movement onset, PMd activity related to the chosen option reaches a peak $\sim 280 \mathrm{~ms}$ before movement initiation, whereas M1 activity follows with a peak $\sim 140 \mathrm{~ms}$ before movement onset. These peaks of activity cannot be related to saccade initiation because, in most trials (74\%-79\%), both monkeys were already looking at the selected target long before the time of the peak (Thura et al., 2014). We have previously proposed that this peak of activity reflects the monkey's volitional commitment to an action choice (Thura and Cisek, 2014).

Figure $9 A$ illustrates activity aligned on movement for the example PMd and M1 cells described above in Figure 5. For both cells, PT-related activity reaches a peak before movement, whereas OT-related activity is suppressed. As shown in Figure 5, during deliberation the activity of both cells was strongly amplified in the fast blocks. However, when aligned on movement onset, we observe that both cells reach a similar peak of activity 
A

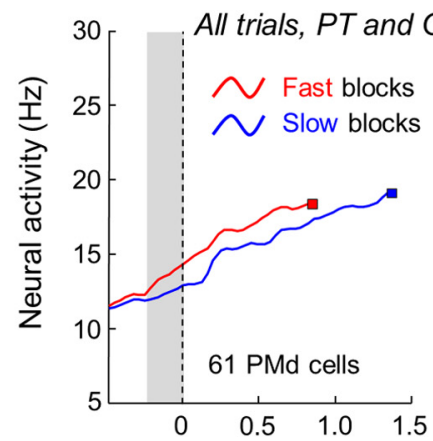

B

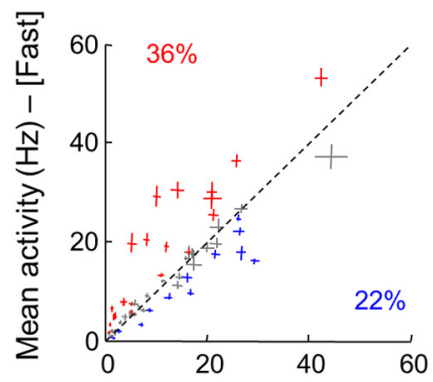

Easy

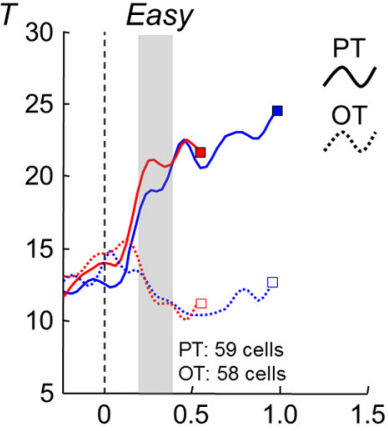

Tim

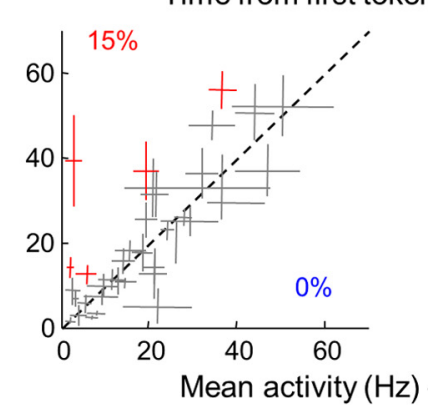

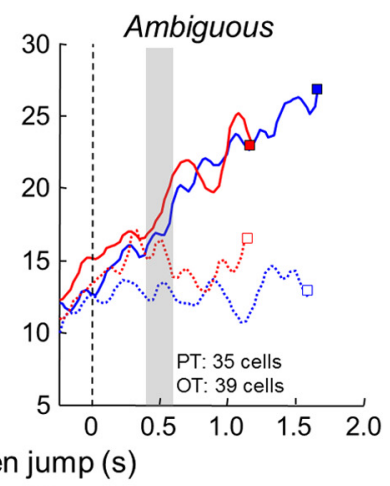

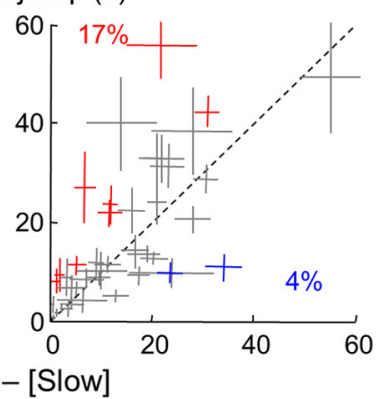

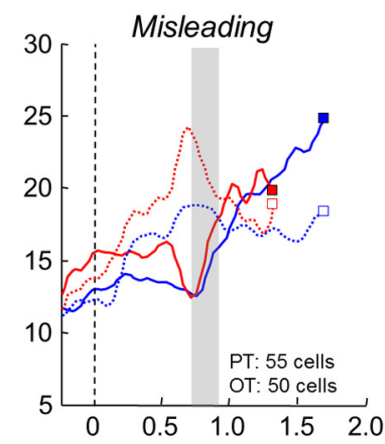

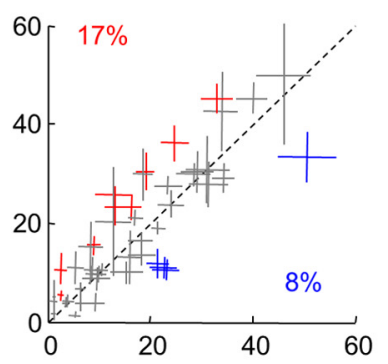

C

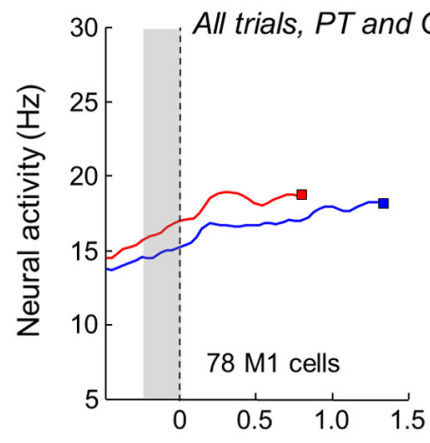

D

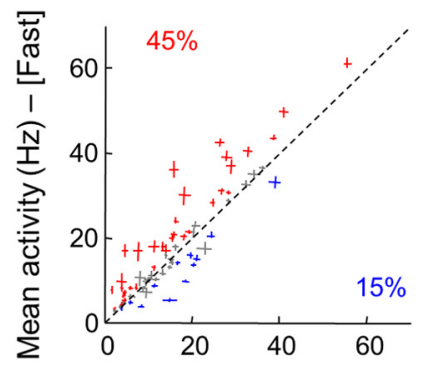

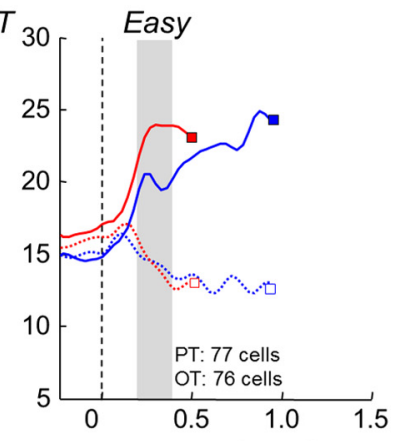

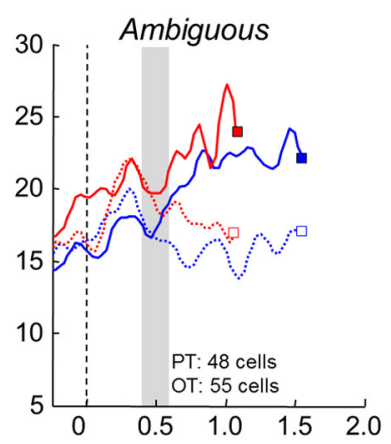

Time from first token jump (s)

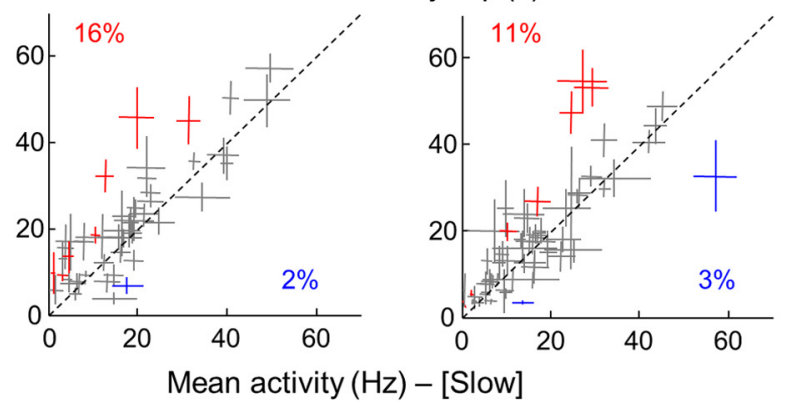

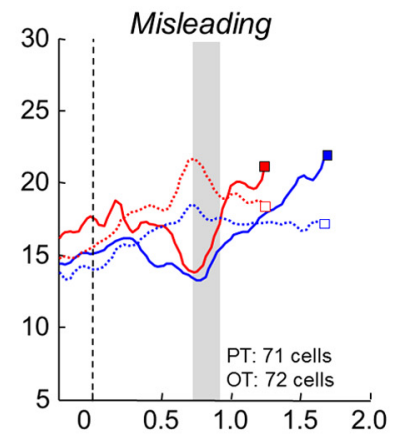

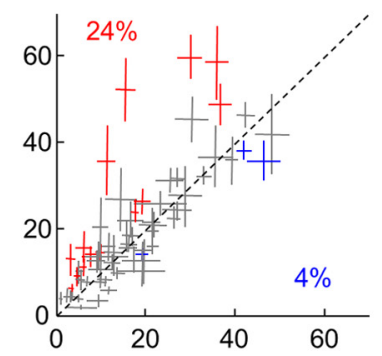

Figure 6. A, Left, Average activity of 61 "decision-related" PMd cells aligned on the firsttoken jump in all trials during the fastblocks (red) or slow blocks (blue). In each block, activity is truncated $280 \mathrm{~ms}$ before movement onset (squares), and both error and correct trials are included. Second panel from left, Average activity of "decision-related" PMd cells during easy trials in which monkeys correctly chose the cells' PT (solid curves, $n=59$ ) or 0 T (dotted curves, $n=58$ ) in the slow blocks (blue) or the fast block (red). To be included, cells had to be recorded during at least 5 trials per condition. Third panel from left, Same as panel 2 for 35 (PT) or 39 (OT) "decision-related" PMd cells recorded during at least 5 ambiguous trials. Right, Same as panel 2 for 55 (PT) or 50 (0T) "decision-related" PMd cells recorded in at least 5 misleading trials. $B$, Left, Comparison of mean neural activity ( \pm SE) of 61 PMd (top) recorded during all trial types and for both OT and PT in the slow block (abscissa) versus the fast block (ordinate) in a $200 \mathrm{~ms}$ period preceding the first token jump ( $A$, left, shaded area). Colored crosses represent neurons with a significant modulation of activity. Gray crosses represent nonsignificant modulations. Red represents stronger activity in the fast blocks. Blue represents stronger activity in the slow blocks. Percentages indicate the proportion of significantly modulated cells. Second panel from left, Same as left panel for 40 PMd neurons during easy trials. Activity is averaged from 200 to $400 \mathrm{~ms}$ after the first token jump ( $A$, panel 2 , shaded area). Here, only trials in which the monkey chose the cell's PT with DT $>400 \mathrm{~ms}$ are included. Third panel from left, Same as left panel for 47 PMd neurons during ambiguous trials. Activity is averaged from 400 to $600 \mathrm{~ms}$ after the first token jump ( $A$, panel 3 , shaded area). Here, trials in which the monkey chose the cell's PT or OT with DT $>600 \mathrm{~ms}$ are included. Right, Same comparison as left panel for the $52 \mathrm{PMd}$ neurons during misleading trials. Activity is averaged from 700 to $900 \mathrm{~ms}$ after the first token jump $(A$, panel 4 , shaded area). Here, only trials for which the monkey chose the cells' 0 T with DT $>900 \mathrm{~ms}$ are included. C, Same as $\boldsymbol{A}$ for a population of decision-related M1 cells (see numbers on the plots). $\boldsymbol{D}$, Same as $\boldsymbol{B}$ for the same population of decision-related cells in M1 (see text for numbers).

regardless of the block. This suggests that the setting of the SAT does not affect the neural threshold that determines commitment to a choice, although it influences the neural activity that leads to the crossing of that threshold.
Consistent with this proposal, the average population activity of both PMd and M1 cells aligned on movement onset is indeed very similar in the two blocks of trials (Fig. 9B). Nevertheless, when we look at the effect of block on individual PMd cells during 
a $200 \mathrm{~ms}$ period around the average peak time (280 ms before movement), we do not observe an absence of the block effect for all cells: 33 PMd cells (54\%) were significantly modulated (WMW test, $p<$ 0.05 ), showing either a stronger (20 of 33 , $61 \%$ ) or a weaker peak activity in fast blocks compared with slow blocks (Fig. $9 C$, top). These balanced modulations are observed despite the fact that RTs were almost always shorter in fast blocks compared with slow blocks in the corresponding recording sessions (Fig. 2B). We conducted the same analysis in M1, comparing activity in a $200 \mathrm{~ms}$ period around the average peak time (140 ms before movement). We also observed a significant proportion of modulated (32 of 78, $41 \%$ ) cells, with a balance between increased activity in the fast (59\%) or slow blocks (Fig. 9C, bottom). Again, RTs during these recording sessions were almost always shorter in the fast than the slow blocks (Fig. 2B).

\section{Time-related neural activity in PMd} and $\mathrm{M} 1$ at decision commitment

The results described above suggest that a context-dependent, time-related signal influences the neural activity responsible for choice deliberation and commitment in PMd and M1. So far, we only looked at a very specific population of "decisionrelated" cells: those that are significantly tuned during the deliberation process. But how global is this time-related signal? Does it also influence cells that are not necessarily involved in the deliberation and commitment process? To examine this, here we consider all of the PMd and M1 cells we recorded in the two blocks of trials (PMd, $n=211$; M1, $n=175$ ), including "decision-related" cells as well as cells that are not significantly tuned before decision commitment. To assess the effect of time on cells' activity, we calculated for each of them a trial-by-trial coefficient of correlation (Pearson product-moment correlation coefficient $r$ ) between DD and the average firing rate in a $200 \mathrm{~ms}$ period just before choice commitment (480-280 ms before movement onset). We found that $30 \%$ of PMd (63 of 211 ) and $30 \%$ of M1 (53 of 175) cells were significantly modulated by DD (Fig. 10A,B). These modulations could be either positive (the longer the decision, the higher the firing rate) or negative. Among such time-dependent cells, some belong to the decision-related cells described above, with a significant modulation of activity by sensory evidence ( 18 of $211,9 \%$ in PMd; 25 of $175,14 \%$ in M1). According to the scatter plot in Figure $10 A, B$, it appears that effects of time and evidence are distributed among a continuum of cells in both PMd and M1. Figure 10C illustrates an example cell recorded in PMd whose activity is strongly modulated by sensory evidence, but not by elapsing time, whereas Fig-
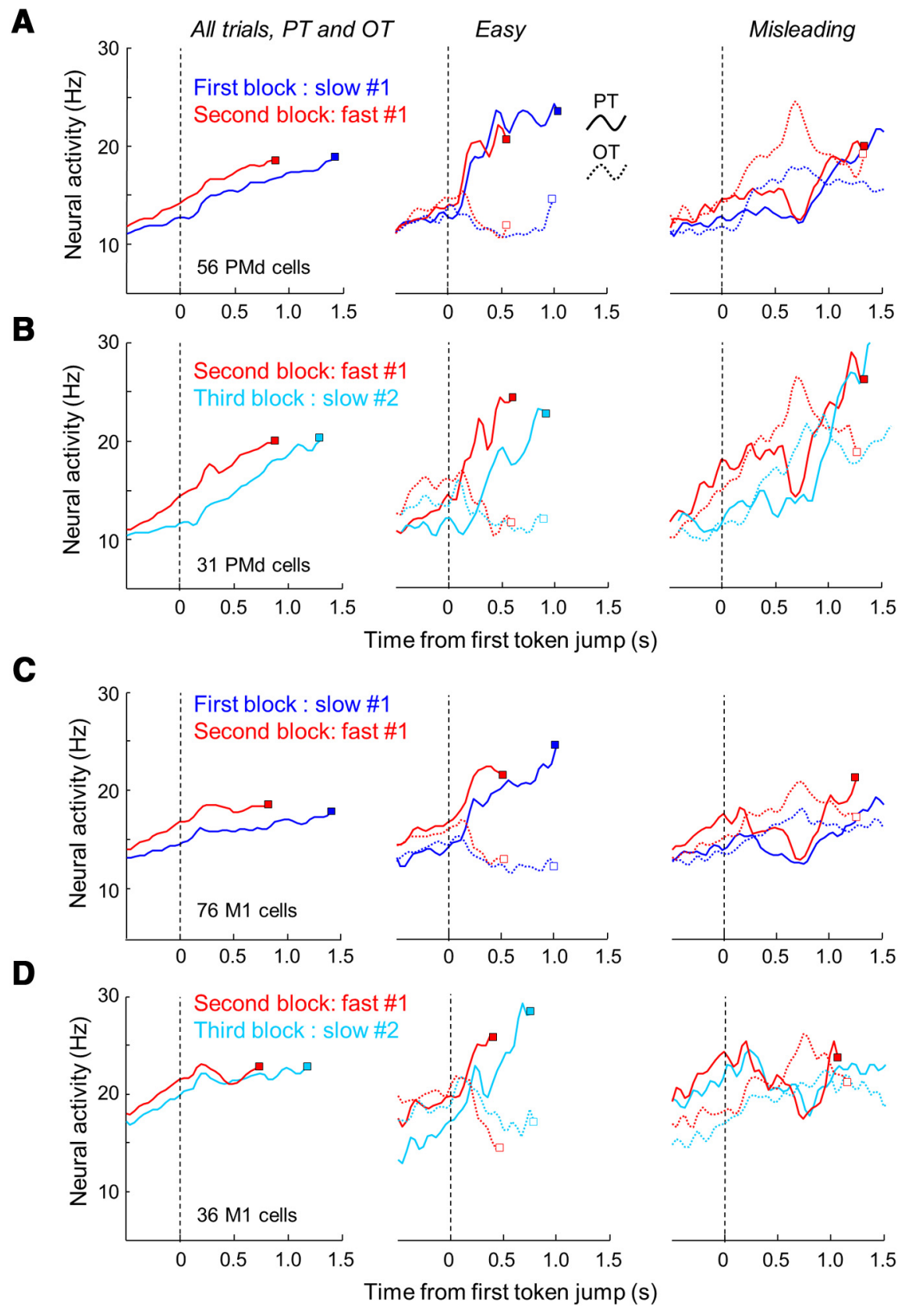

Figure 7. A, Left, Average activity of 56 PMd "decision-related" cells aligned on the first token jump in all trials during the fast blocks (red) or slow blocks (blue). Here, we only include slow block trials performed when the slow block preceded the fast block. Same convention as in Figure 6 A. Middle, Same as left panel for PMd neurons recorded during easy trials. Right, Same as left panel for PMd neurons recorded during misleading trials. $\boldsymbol{B}$, Same as $\boldsymbol{A}$, but here we only include slow block trials (light blue) from a second slow block, performed after a fast block (red). $\boldsymbol{C}, \boldsymbol{D}$, Same analyses as in $\boldsymbol{A}$ and $\boldsymbol{B}$ for a population of decision-related $\mathrm{M} 1$ cells.

ure $10 D$ shows a PMd cell less modulated by sensory evidence, but strongly affected by elapsing time.

Does this influence of DD reflect only elapsing time or is it also context dependent, like the putative urgency signals shown in Figure $3 B$ ? For these time-related activities, we can assess the level of activity preceding DT and compare it between the two blocks. If this time-dependent signal is indeed related to the urgency signal of our decision mechanism, we should observe stronger activity after longer DDs regardless of the blocks (because urgency increases with time in both conditions) and higher activity in the fast block compared with the slow block, especially for the shortest decisions. While the evidence-related cell (weakly af- 
A

PMd, 30 modulated cells
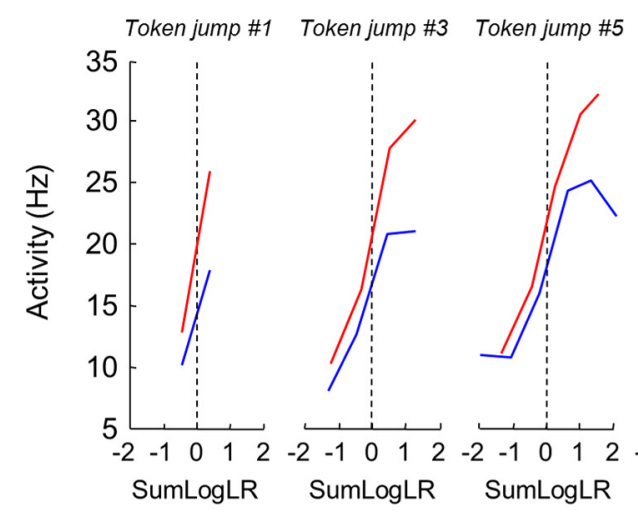

$\sim$ Slow blocks

$\sim$ Fast blocks

C

M1, 47 modulated cells
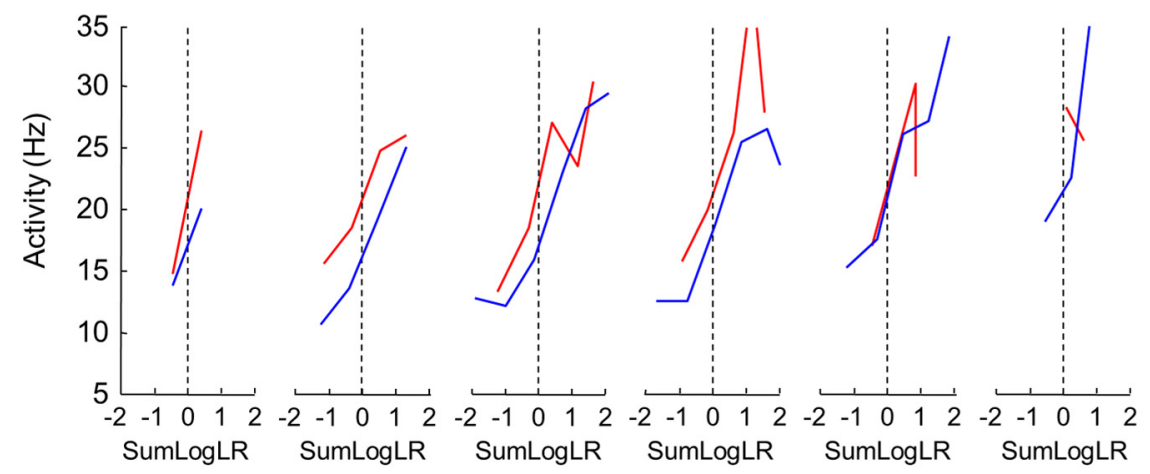

B

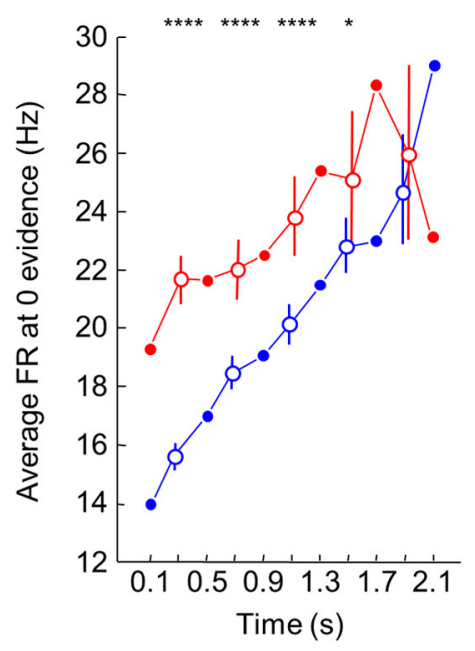

D

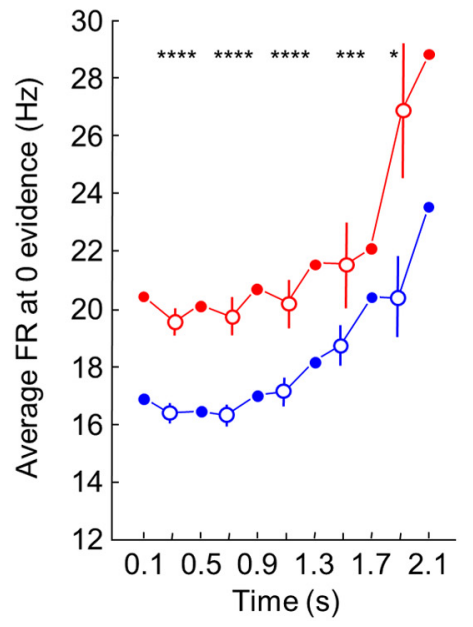

Figure 8. A, Evolution of the relationship between sensory evidence and the average PMd activity during the slow blocks (blue) or the fast blocks (red). To be included, cells had to have significantly higher activity in fast blocks in at least one of the tested periods described in Figure $6(n=30)$. For both blocks, lines indicate the relationship between the SumLogLR calculated with respect to the cells' PT and the mean neural activity calculated $200 \mathrm{~ms}$ later in a $200 \mathrm{~ms}$ epoch. Only epochs preceding our estimated DT are included. For clarity, this relationship is only illustrated for token jumps 1, 3, 5, 7, 9 and 11. $B$, Evolution of the averaged activity ( \pm SE) of the PMd population $(n=30)$ calculated for the condition when SumLogLR $=0$ (the evidence is equal for each target) as a function of time in either the slow (blue) or the fast blocks (red). For odd-numbered jumps, the firing rate at zero evidence was calculated by interpolation (filled circles). For even-numbered jumps, vertical bars represent the Cls around the means calculated through a bootstrap procedure (see Materials and Methods). ${ }^{* * *} p<0.0001$. ${ }^{* * *} p<0.001$. ${ }^{* *} p<0.01 .{ }^{*} p<$ 0.05. C, Same as $\boldsymbol{A}$ for the population of M1 cells significantly and positively modulated by the block in at least one of the tested periods described in Figure $6(n=47)$. D, Same as $\boldsymbol{B}$ for the M1 cells.

fected by DD) shows only a weak trend of time-related modulation that is not context-dependent (Fig. 10E), the pattern of activity of the time-related cell strongly supports our hypothesis (Fig. 10F). It shows a strong context-dependent effect: for the same DD range, activity is higher in the fast condition compared with the slow condition, particularly for the shortest decisions.

Next, we tested this prediction at the population level, following the same logic: if the population of time-sensitive cells are influenced by the urgency signals estimated from monkeys' behavior (Fig. 3B), then we should observe three trends for those cells that are positively correlated with DD: (1) activity at DT should increase with DD, regardless of the block; (2) activity preceding short decisions in the fast blocks will be higher than activity preceding short decisions in the slow trials; but (3) activity should be similar for the longest decisions in the two blocks because the urgency signal tends to converge in the two blocks for long decisions (Fig. 3B). Furthermore, we should observe the opposite pattern for cells that are negatively correlated with DD.

We performed two analyses to test these predictions at the population level. First, we took advantage of the large variability of animals' RTs in the tokens task (Monkey S: 274-2773 ms; SD: $503 \mathrm{~ms}$; Monkey Z: 249-2851 ms; SD: $583 \mathrm{~ms}$ ) to divide the trials (for each cell) into two equal size groups based on the median $\mathrm{DD}$, regardless of the block condition. Figure $11 B, G$ illustrates for each group of trials performed in both blocks the average firing rate aligned on movement onset across all PMd (top panels) and M1 (bottom panels) cells, which were either positively (Fig. 11B) or negatively (Fig. 11G) correlated with DD in a $200 \mathrm{~ms}$ epoch preceding decision commitment (Pearson coefficient of correlation, $p<0.05$ ). These plots show that PMd and M1 timesensitive cells appear to reflect the influence of the contextdependent urgency signals estimated from monkeys' behavior: neural activity related to the shortest decisions tends to be higher (lower) in the fast blocks compared with the slow blocks for cells that are positively (negatively) correlated with DD, whereas activity related to the longest decisions tends to be similar in both blocks. To control for the possibility that these effects are driven by neurons with high firing rates, we performed a normalization of each cell activity (based on the activity of each cell around commitment time) before averaging across populations. The re- 
A
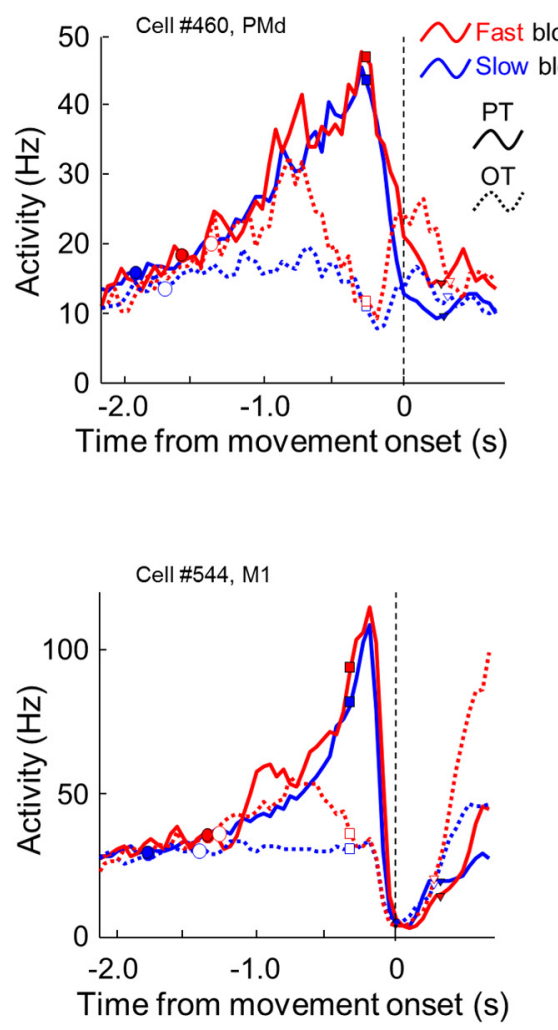

B

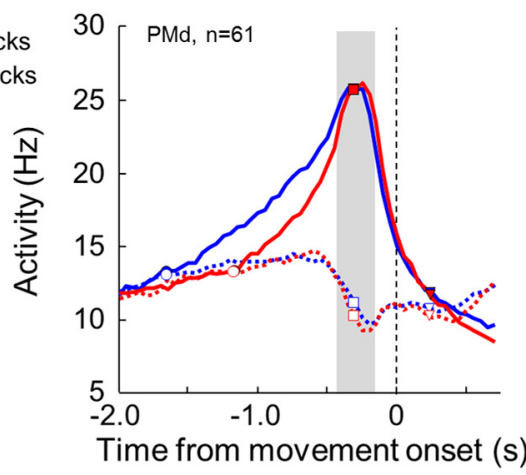

C
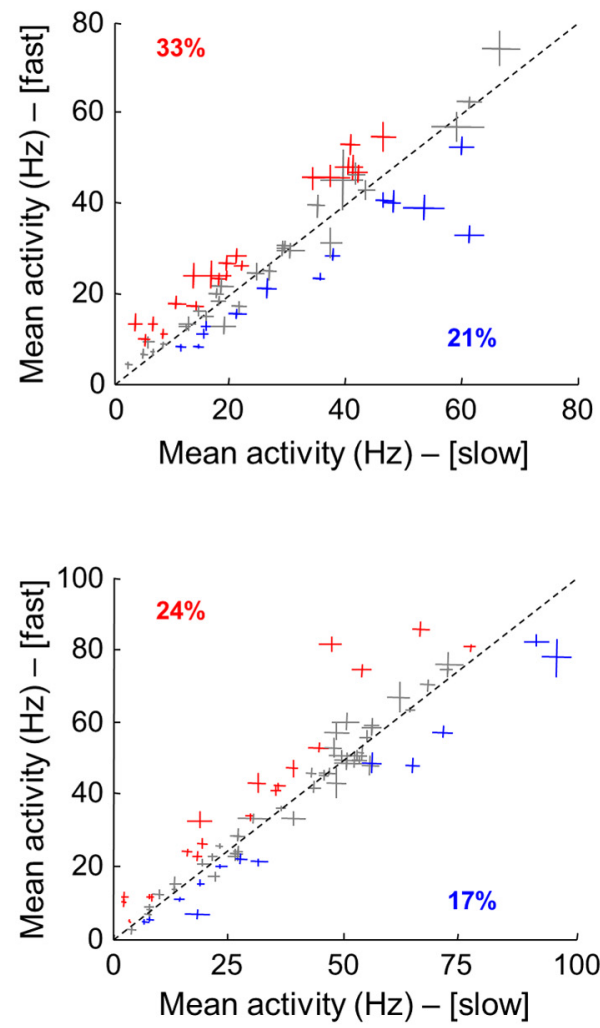

Figure 9. A, Top, Activity of the PMd cell shown in Figure 5C,D, aligned on movement onset. Trials in which the monkey chose the cell's PT (solid lines) or OT (dotted lines) are plotted for the slow (blue) or fast blocks (red). Trials collected in the two sets of slow blocks are merged. Circles represent the first token jump. Squares represent estimated DT. Triangles represent movement offset. Bottom, Same as top panel for the M1 cell shown in Figure $5 A, B$. Trials collected in the two sets of slow and fast blocks are merged. $\boldsymbol{B}$, Average neural responses of the 61 PMd (top) and the $78 \mathrm{M} 1$ (bottom) decision-related cells aligned on movement onset when monkeys either chose the cells' PT (solid curves) or OT (dotted curves) in the slow (blue) or fast blocks (red). $C$, Comparison of the PT-related activity of each of the 61 PMd and $78 \mathrm{M} 1$ decision related cells in a 200 ms period surrounding the population peak activity ( 280 ms before movement in PMd, 140 ms before movement in M1; gray shaded areas). Same conventions as in Figure 6B-D.

sults (data not shown) were qualitatively indistinguishable from those obtained when the raw firing rate was considered.

As illustrated in Figure 11A, F, monkeys' behavior was highly consistent during sessions in which these cells were recorded: average sensory evidence at commitment time was higher in slow blocks compared with fast blocks, especially for the shortest decisions, suggesting that urgency was initially lower but grew faster in slow blocks compared with fast blocks. To better demonstrate this link between behavior and neural activity, we calculated for each group of trials and in each block the average firing rate of the cells in the $200 \mathrm{~ms}$ period preceding our estimate of DT and we then averaged this activity across all the PMd or M1 timemodulated cells. The results are illustrated in Figure $11 C, H$. In PMd, activity is significantly modulated by DD in the slow blocks, both for the positively and negatively correlated cells ( $17 \mathrm{vs} 26 \mathrm{~Hz}$, CI from bootstrap test, $p<0.0001$ and 19 vs $14 \mathrm{~Hz}, p<0.0001$, respectively), whereas the effect of duration is weaker in the fast blocks but still very significant. The effect of block is stronger $(p<0.0001)$ for the shortest decisions than for the longest ones, for both PMd populations, as predicted by the urgency model. In M1, we found similar qualitative results for both cells positively and negatively correlated with DDs. Overall, these data are consistent with the hypothesis that activity is influenced by the urgency signals estimated from behavior (Fig. 3B).

Next, we performed a within-block correlation analysis between DD and firing rate in a $200 \mathrm{~ms}$ window preceding decision commitment (illustrated in two example cells in Fig. 10E,F) for each of the PMd and M1 cells positively or negatively correlated with DD. As expected, we found a significant correlation between activity and DD in all cells when the analysis is conducted in the slow blocks (this trivial significant correlation was indeed the criterion for including a cell in this analysis). The percentage of cells showing a significant correlation between these two variables was lower in the fast condition. More interestingly, we found that, in both areas, neural activity before commitment was modulated as predicted by the UGM: for the positively (negatively) correlated cells, the regression slopes are usually positive (negative) and steeper in the slow blocks and intercepts are higher (lower) in the fast blocks (Fig. 11 D,E for the PMd and M1 positively correlated cells; Fig. $11 \mathrm{I}, J$ for the negatively correlated cells).

\section{Velocity-related neural activity in PMd and M1 before movement execution}

Finally, we looked at another type of cell, usually not significantly tuned during the deliberation process but tuned just before movement onset. The premovement activity in these PMd and M1 cells is occasionally correlated with the speed of the upcoming reaching movement (Moran and Schwartz, 1999; Churchland et al., 2006). Figure $12 \mathrm{~A}$ illustrates an example speed-sensitive cell (correlation between reach peak velocity and PT-related activity in the $300 \mathrm{~ms}$ epoch preceding movement onset, $r=0.47, p<$ 0.001 ) recorded in M1. Interestingly, when sorted as a function of $\mathrm{DD}$, the speed-related premovement burst is stronger after longer 
A

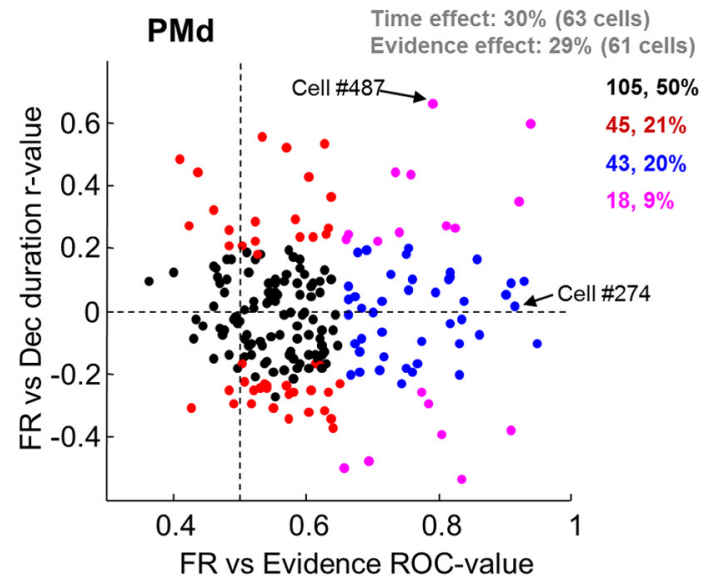

B

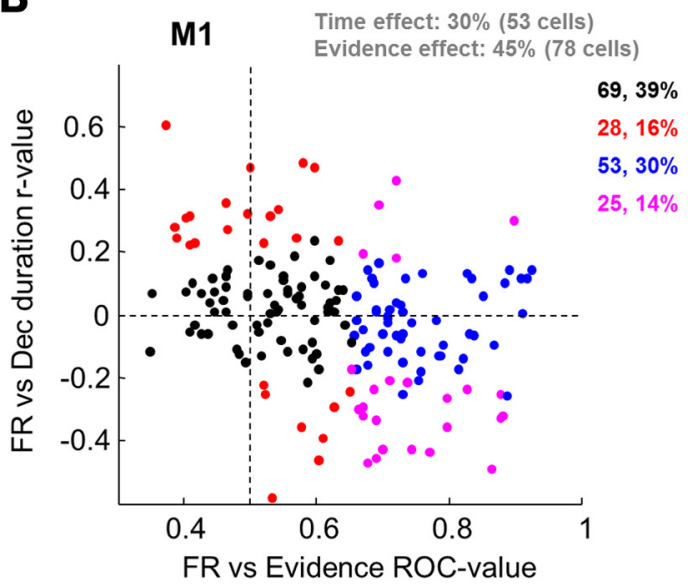

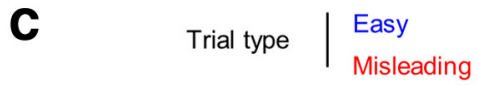

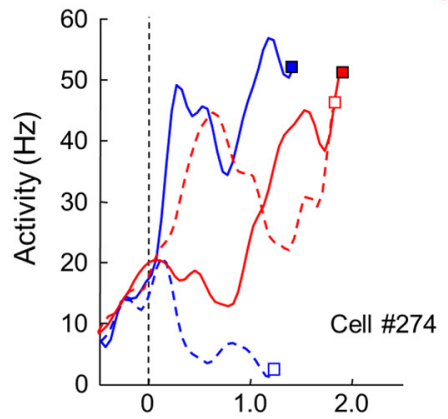

D

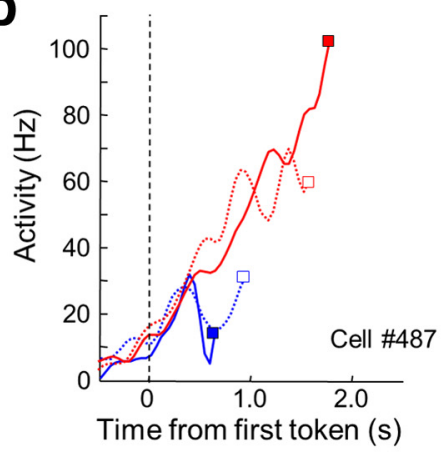

\begin{tabular}{l|l} 
Trial duration & $\begin{array}{l}\text { Short } \\
\text { Medium } \\
\text { Long }\end{array}$
\end{tabular}
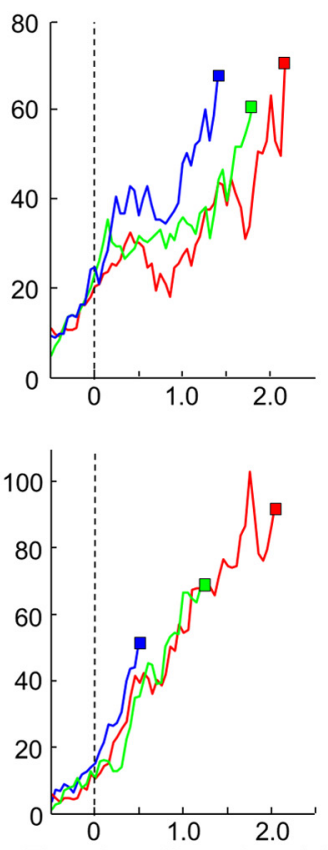

Time from first token (s)
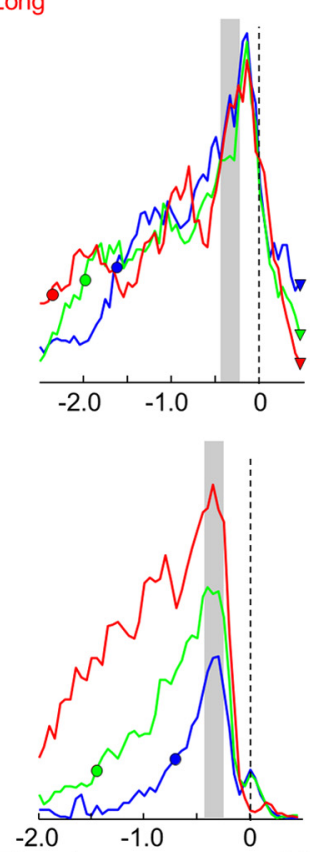

s) Time from movement (s)
E Block $\mid \begin{aligned} & \text { Slow } \\ & \text { Fast }\end{aligned}$

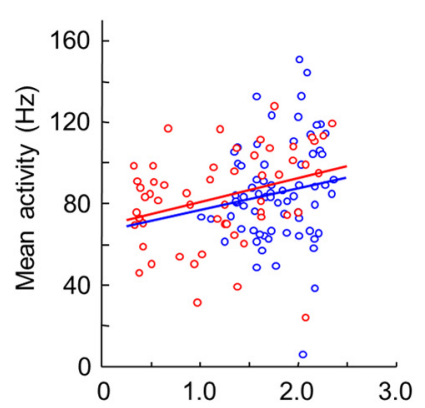

$\mathbf{F}$

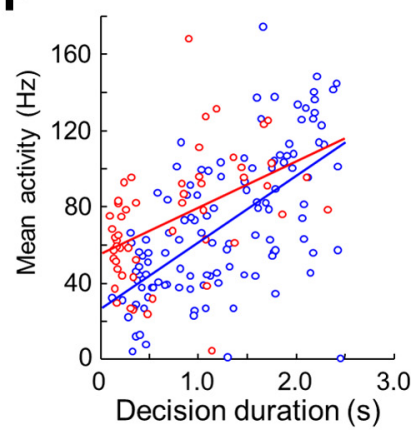

Figure 10. A, Scatter plot showing, for the 211 PMd cells, the $r$ values of the correlation between average activity in a 200 ms period preceding commitment and DD (ordinate) against the ROC score related to the effect of sensory evidence on activity (abscissa). Each dot represents a cell. Color code corresponds to statistical effects of the two factors: evidence and DD. Blue dots represent cells only sensitive to sensory evidence (i.e., significantly tuned before DT, ROC $>0.65$ ). Red dots represent cells significantly modulated by DD (Pearson correlation coefficient $r, p<0.05$ ). Magenta dots represent cells modulated by both evidence and DD. Black dots represent cells unaffected by these two factors. B, Same as $A$ for the population of 175 M1 cells. C, Top left, Activity of a sensory evidence-related PMd cell recorded in the tokens task during easy (blue) and misleading (red) trials when the monkey chose either the cell's PT (solid curves) or OT (dashed curves). Activity is aligned on the first token jump, and only trials performed in the slow block are illustrated here. Middle, Activity of the same cell aligned on the first token jump, averaged across 3 groups of trials grouped by DD, the shortest (blue), the medium (green), and the longest decisions (red) performed in the slow block. Activity is truncated 280 ms before movement onset (squares). Right, Same as middle, but here activity is aligned on movement onset. Circles represent the first token jump. Triangles represent movement offset. D, Same as ( for another PMd cell, which is related to DD. $E$, Mean activity of the PMd cell shown in C, calculated in a $200 \mathrm{~ms}$ period preceding estimated DT (C, right, shaded area) as a function of DD in either the slow block (blue) or the fast block (red). Each dot represents data from a single trial. Solid lines indicate a linear regression calculated through the data of each block. $\boldsymbol{F}$, Same as $\boldsymbol{E}$ for the PMd cell related to DD depicted in $\boldsymbol{D}$.

decisions (correlation between DD and PT-related activity in the $300 \mathrm{~ms}$ epoch preceding movement onset, $r=0.47, p<0.001$ ). Consistent with the assumption that this burst influences the upcoming movement speed, we observed that the longest decisions tend to lead to the fastest movements $(r=0.4, p<0.05$; Fig. 12B).

In a previous report (Thura et al., 2014), we showed that the reaching movements executed by the monkeys to report their decisions were shorter in duration after longer decisions. We also showed that this effect was block dependent, as the range of the duration reduction was bigger in the slow blocks of trials than the fast blocks and the difference was larger for the shortest decisions. Because this pattern is again reminiscent of the urgency signals estimated from choice behavior, we hypothesized that the same urgency signal that pushes animals to make their decision also influences how they execute their reaching movements to report these decisions (for more details, see Thura et al., 2014). 
A
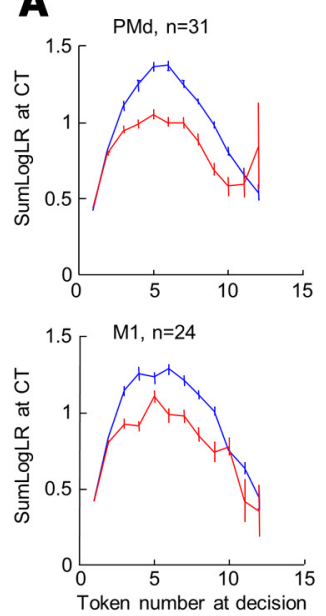

F
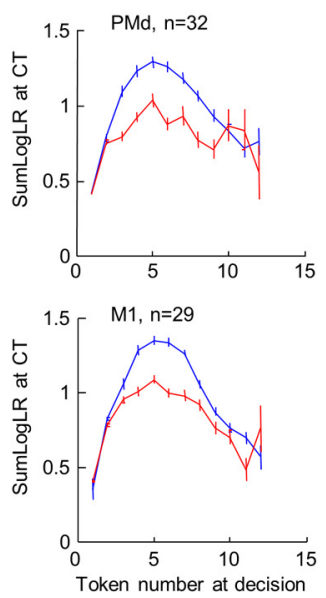

B
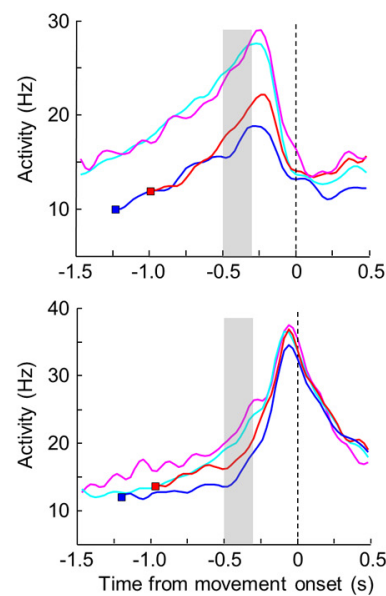

G
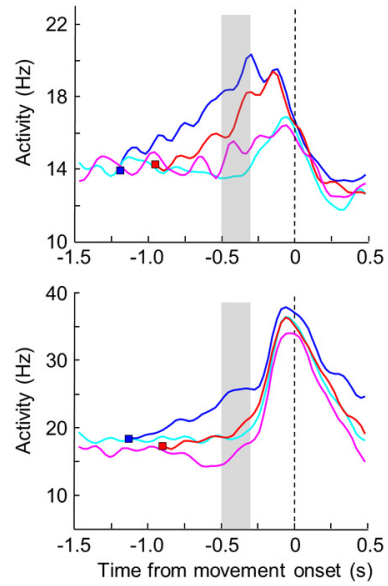

C
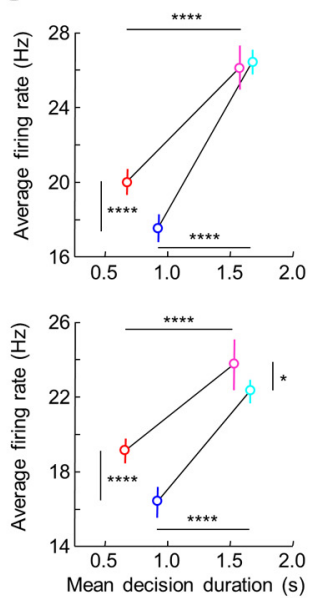

H
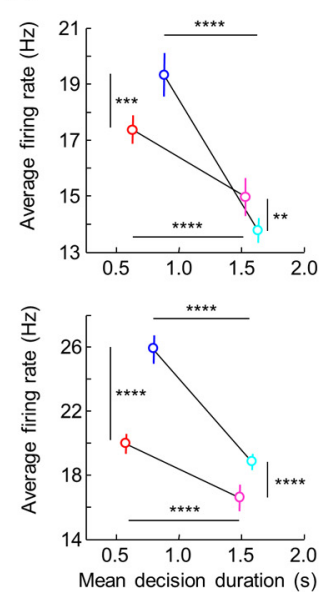

D

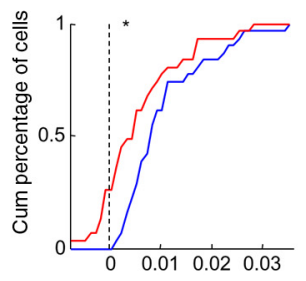

E
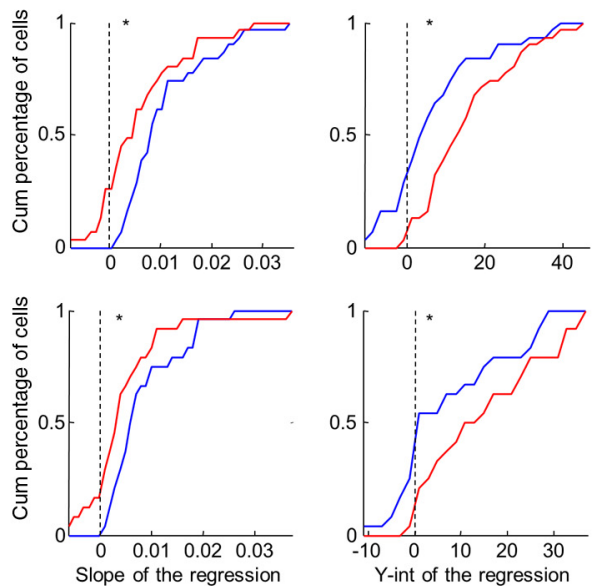

J
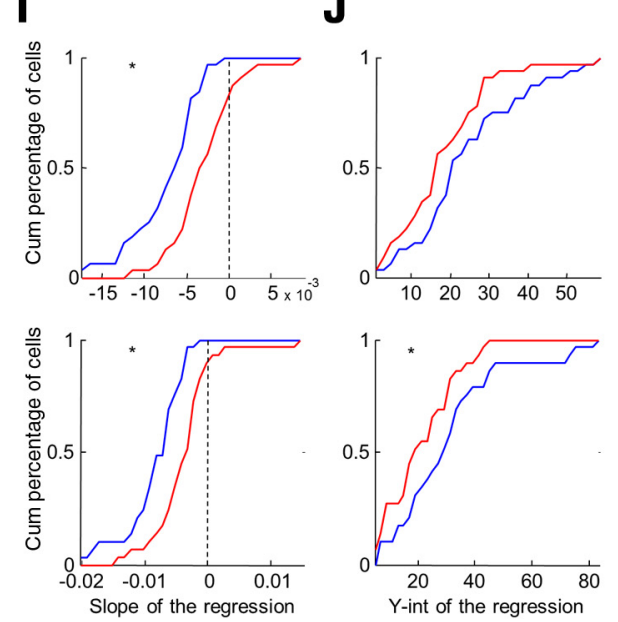

Figure 11. A, SumLogLR curves calculated in the slow and fast blocks for the trials during the recording of the $31 \mathrm{PMd}$ (top) and the $24 \mathrm{M1}$ (bottom) cells significantly and positively modulated by DD (in a $200 \mathrm{~ms}$ period preceding decision commitment). B, Average response of the $31 \mathrm{PMd}$ (top) and the $24 \mathrm{M} 1$ cells as a function of DD and block, aligned on movement onset. For each cell, the median DD of all trials (performed in both the slow and the fast block) is calculated and trials within each block are then split into two groups based on this median. The mean activity in each group (dark blue represents the shortest decisions of the slow blocks; dark red represents the shortest decisions of the fast blocks; light blue represents the longest decisions of the slow blocks; light red represents the longest decisions of the fast blocks) is then aligned on movement onset and averaged across all cells. C, Mean PMd (top) or M1 activity of each group of trials calculated in a $200 \mathrm{~ms}$ period preceding DT ( $\boldsymbol{B}$, gray shaded area) plotted as a function of the mean DD of the corresponding groups. Vertical bars represent $C$ ls around the means computed through a bootstrap procedure. ${ }^{* * * *} p<0.0001 .{ }^{* * *} p<0.001 .{ }^{* *} p<0.01 .{ }^{*} p<0.05$. D , Cumulative distributions of the slope values computed from a trial-by-trial linear regression analysis performed in each of the 31 PMd (top) and $24 \mathrm{M} 1$ cells between neural activity in a $200 \mathrm{~ms}$ window preceding decision commitment and DD in either the slow (blue) or fast block (red). Asterisks indicate a significant difference between the distributions (WMW test, $p<0.05$ ). $\boldsymbol{E}$, Cumulative distributions of the $y$-intercept values computed from this same trial-by-trial linear regression analysis in each of the 31 PMd (top) and $24 \mathrm{M} 1$ cell in either the slow (blue) or fast block (red). Asterisks indicate a significant difference between the distributions (WMW test, $p<0.05$ ). $\boldsymbol{F}-\boldsymbol{J}$, Same as $\boldsymbol{A}-\boldsymbol{E}$ for a population of 32 PMd and $29 \mathrm{M} 1$ cells significantly but negatively modulated by DD (in a $200 \mathrm{~ms}$ period before commitment).

The manner in which monkeys reduced the duration of movements was complex and idiosyncratic. In brief, Monkey S mainly increased movement speed as time passed and also increased speed in the fast blocks compared with slow blocks. In contrast, Monkey $\mathrm{Z}$ used a combination of increased speed and reduced amplitude to reduce movement duration after longer decisions. Nevertheless, in all cases, the speed of movements increased with $\mathrm{DD}$, as if the vigor of actions is related to the urgency at the time the decision is made. The activity of the speed-related cell depicted in Figure $12 \mathrm{~A}$ is consistent with this proposal.

To assess this hypothesis at the population level, we selected all of the PMd and M1 cells that show a significant and positive correlation between movement speed and the firing rate in a 300 $\mathrm{ms}$ period preceding movement onset (Pearson coefficient of correlation, $p<0.05$ ). For each of these cells and regardless of the block, we calculated the median RT that divides the trials in two equal-size groups. Finally, we plot for each block the average activity of the speed-sensitive cells in the two categories of trials: the shortest versus the longest decisions.

In Monkey S (Fig. 12C,D), we found that, despite the low number of trials corresponding to the $10 \mathrm{PMd}$ and $9 \mathrm{M} 1$ speedsensitive cells, movements following the longest decisions were faster compared with those that followed the shortest decisions, especially in the slow blocks of trials (CIs from bootstrap, $p<$ 0.0001 ). In the fast blocks, the increase of movement speed with DD was smaller but still significant $(p<0.001$ and $p<0.05$ for the sessions during which PMd and M1 have been recorded in Monkey S, respectively). As predicted by the UGM, we found that movements following the shortest decisions were significantly faster when performed in the fast blocks than in the slow blocks (Fig. 12C, $D$, CIs computed from a bootstrap test; $p<0.0001$ ). This is in agreement with our observations based on the large behavioral dataset (Thura et al., 2014) and in agreement with the proposal that the context-dependent urgency signal that deter- 
A

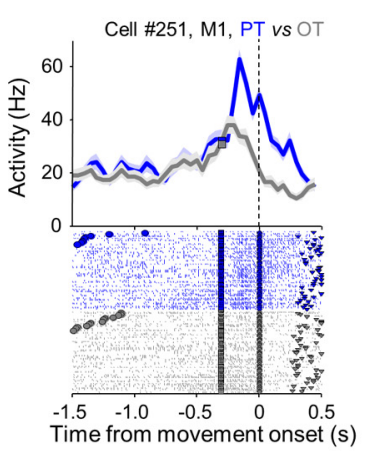

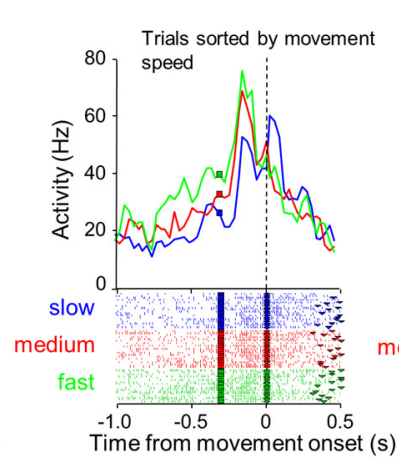

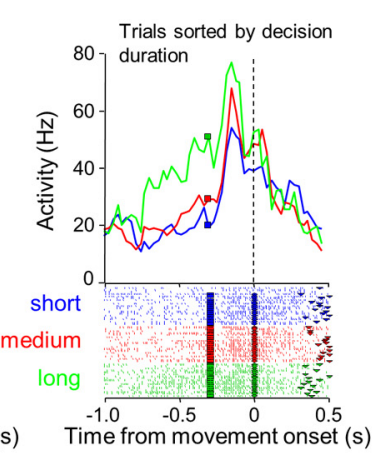

B

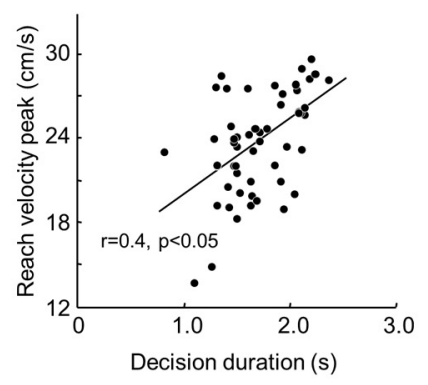

C

Monkey S, PMd
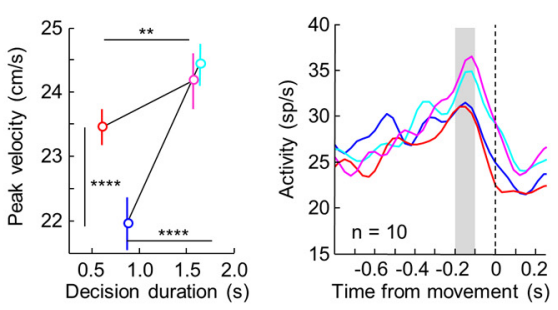

\section{$\mathbf{E}$}
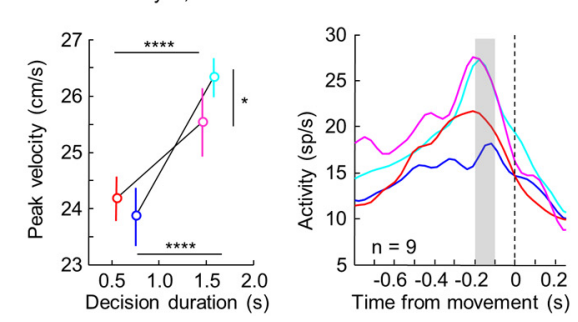

D

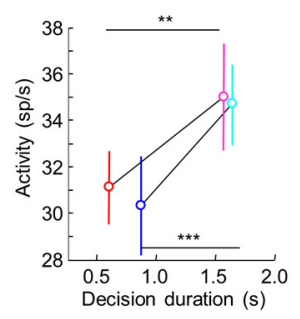

\section{Monkey S, M1}
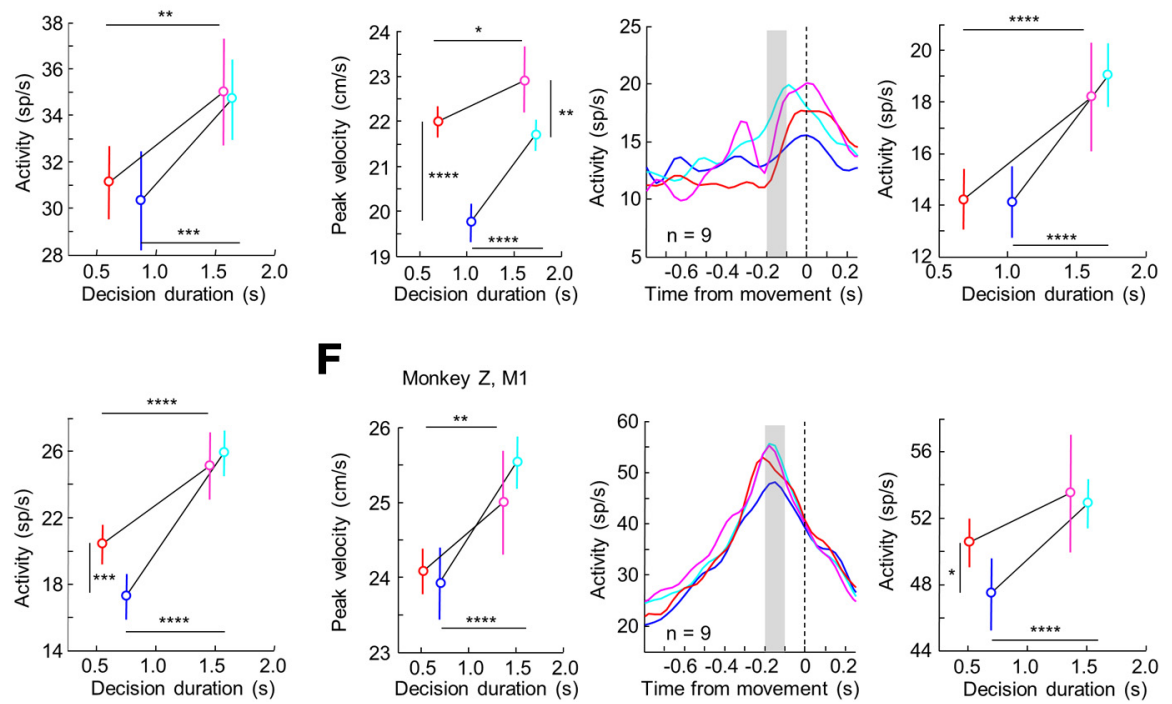

$\mathbf{F}$

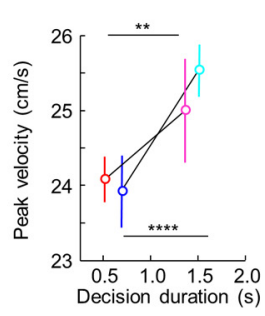

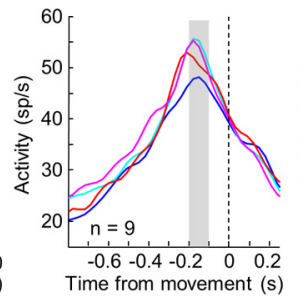

Figure 12. A, Left, Activity of an M1 cell recorded in the tokens task when the monkey chose the cell's PT (blue) or 0T (gray). Activity is aligned on movement onset and illustrated with spike density functions (top) and rasters (bottom), sorted by DD. Circles represent the first token jump. Squares represent DT. Triangles represent movement offset. Middle, Activity of the same cell with PT-related trials sorted as a function of peak movement speed (blue represents slowest; red represents medium; green represents fastest). Right, Activity of the same cell with PT-related trials sorted as a function of DD (blue represents shortest; red represents medium; green represents longest). $\boldsymbol{B}$, Trial-by-trial correlation between DD and peak movement speed for PT trials of the cell depicted in $\boldsymbol{A}$. Black line indicates a linear regression through the data. C, Left, Mean movement peak velocity as a function of mean DD calculated from trials during which 10 PMd cells significantly correlated with movement speed were recorded in Monkey S. For each cell, the median DD of all trials (performed in both the slow and the fast block) was calculated and trials of each block were divided into two groups: the shortest and the longest decisions. The mean velocity peak within each group (dark blue represents the shortest decisions of the slow blocks; dark red represents the shortest decisions of the fast blocks; light blue represents the longest decisions of the slow blocks; light red represents the longest decisions of the fast blocks) was then averaged across these cells. Vertical bars represent $\mathrm{Cl}$ around the means computed through a bootstrap procedure. ${ }^{* * *} p<0.0001$. ${ }^{* * *} p<0.001 .{ }^{* *} p<0.01 .{ }^{*} p<0.05$. Middle, Average response of the 10 PMd speed-modulated cells in each of the four groups defined based on DD. Right, Mean activity calculated in a period from 200 to $100 \mathrm{~ms}$ preceding movement onset (gray shaded area). Vertical bars represent $\mathrm{Cls}$ around the means computed through a bootstrap procedure. D, Same as C for a population of $9 \mathrm{M} 1$ cells modulated by movement velocity and recorded in Monkey S. E, Same as C for 9 PMd speed-modulated cells recorded in Monkey Z. F, Same as $\boldsymbol{E}$ for 9 M1 speed-modulated cells recorded in Monkey Z.

mines DTs also influences how Monkey S executes his movements. The neural recordings presented in the current paper are consistent with this hypothesis by showing that PMd speedrelated cells have a stronger burst after longer decisions ( 35 vs 31 $\mathrm{Hz}$ in the fast blocks, $p<0.01 ; 35 \mathrm{vs} 30.5 \mathrm{~Hz}$ in the slow blocks, $p<0.001$ ), presumably leading to faster movements. For the 9 M1 cells recorded in Monkey S, firing rate is also significantly stronger after long decisions compared with short decisions, regardless of the block condition $(p<0.0001)$. A block-dependent effect of DD on speed-related activity also appears in Monkey Z. For the M1 cells, the effect of DD is only significant in the slow blocks $(p<0.0001$; Fig. $12 F)$. In these cells, activity was also stronger in the fast blocks compared with the slow blocks for the shortest decisions $(p<0.05)$, but not for the longest, as predicted by the UGM. For the 9 PMd cells recorded in Monkey Z, the effect of DD on activity is significant $(p<0.0001)$ in both the slow and the fast blocks (Fig. 12E). The larger range of the effect of urgency in the slow blocks of trials as well as the amplification of activity in the fast blocks for the shortest decisions $(p<0.001)$ but not for the longest is in agreement with our detailed behavioral data (Thura et al., 2014). However, because of the low number of speed-related neurons we sampled, any conclusions based on these results must be considered preliminary and require additional study.

\section{Discussion}

Reward rate is a major factor motivating animal behavior (Bogacz et al., 2010b; Balci et al., 2011); and to optimize it, animals must adjust their SAT during both decision-making and movement execution (Chittka et al., 2009). SAT adjustment in decisions has generally been discussed in terms of EAMs (Ratcliff, 1978; Bogacz et al., 2010a), in which control of the SAT is equiv- 
alent to controlling the accuracy criterion of the evidence needed for commitment. At the neural level, this could be accomplished through a variety of mechanisms, including changing the neural firing threshold for initiating movement, the baseline activity before evidence processing, or the gain with which evidence is processed (for review, see Standage et al., 2014; Heitz, 2014).

Recently, two studies have examined SAT adjustment at the single-cell level. During a visual search task, Heitz and Schall (2012) found that the instruction to respond slowly reduced the baseline activity of FEF visual cells and delayed the onset and gain of their sensory processing, and also reduced the activity of FEF movement cells. During a motion discrimination task, Hanks et al. (2014) found that, when accuracy was emphasized (by delaying reward delivery), activity in lateral intraparietal area exhibited both a decreased baseline and gain but did not change processing onset time or the activity before saccade initiation. Human neuroimaging studies have also reported that an emphasis on speed increases baseline activity in the striatum, the presupplemental motor area, as well as premotor and parietal regions (Forstmann et al., 2008; Ivanoff et al., 2008; van Veen et al., 2008; Bogacz et al., 2010a; Mansfield et al., 2011; Wenzlaff et al., 2011). These findings make good sense because, with a higher baseline and/or gain, it is easier for neural activity to build up to whatever is the threshold (Hanes and Schall, 1996; Roitman and Shadlen, 2002; Ratcliff et al., 2007) or dynamic attractor (Grossberg, 1973; Wang, 2002; Standage et al., 2011) that determines the commitment to a decision.

A variety of models have been proposed to explain neural activity during decision-making tasks. These include the driftdiffusion model (Ratcliff, 1978; Gold and Shadlen, 2007), which assumes perfect integration of sensory evidence to a fixed accuracy criterion, as well as variations in which the accuracy criterion decreases over time (Drugowitsch et al., 2012) possibly due to a rising urgency signal (Ditterich, 2006; Churchland et al., 2008) or in which integration is "leaky" (Usher and McClelland, 2001). One can conceive of each of these models as lying within a space defined by different assumptions about parameter settings (Thura, 2015). For example, the drift-diffusion model lies at a corner corresponding to zero leak and zero urgency, the leaky competing accumulator (Usher and McClelland, 2001) assumes leak but no urgency, the bounded accumulator with urgency (Drugowitsch et al., 2012) assumes no leak but a growing urgency, and the UGM assumes both a large leak and growing urgency (Cisek et al., 2009; Thura et al., 2012). While there is an ongoing debate as to which model is best (Hawkins et al., 2015a; Hawkins et al., 2015b) and whether the answer may be taskdependent, it is important to note that all of these models permit similar mechanisms for SAT adjustment (Heitz, 2014). In particular, an increase in the baseline and/or gain of neural responses would increase the speed and reduce the accuracy of all these models, regardless of where they lie in the space of parameter settings.

Here, we have used the UGM to interpret our data. The reason is that our previous analyses of behavior and neural activity during the tokens task (from the same animals) have already ruled out other classes of models. In particular, we have shown that neural activity in PMd and M1 quickly tracks the state of sensory evidence without integrating it (Thura and Cisek, 2014) (Figs. 4, $5)$, which is incompatible with the drift-diffusion model and other long-time-constant models. We have also shown that neural activity increases with time even for identical levels of sensory evidence (Thura and Cisek, 2014) (Fig. 3). A short time constant is important in any task in which the sensory information can change at any time, as during natural behavior, and an urgency signal (or some mechanism for reducing the accuracy criterion over time) is optimal for maximizing reward rates in any free response task (Drugowitsch et al., 2012; Gluth et al., 2012; Thura et al., 2012). In the context of the UGM, analyses of behavioral data in our task have suggested that, to adjust the SAT, the animals change the intercept and slope of their urgency signal in a block-dependent manner (Thura et al., 2014). In particular, the signal starts higher but rises more slowly in the fast block than in the slow block (Fig. 3B).

Our neural data are consistent with this proposal. For cells reflecting the deliberation process ("decision cells," 29\% in PMd and $45 \%$ in M1), baseline activity is higher in the fast than the slow block (Figs. 6, 7), and their response to equal sensory evidence is stronger in the fast than the slow block, especially during the early part of trials (Fig. 8). This is also consistent with previous studies. In particular, if the urgency signal modulates how sensory evidence is turned into neural activity, then it would explain both the changes in baseline activity (when the evidence for each of two choices is 0.5 ) as well as the changes in gain after stimulus information is provided, whether or not that gain is time-dependent in a given task (Ditterich, 2006; Cisek et al., 2009; Thura et al., 2012). In other words, despite the significant differences between tasks (static vs changing evidence, instructed vs volitional SAT adjustment), despite the different regions from which neural activity is recorded (frontal vs parietal, skeletal vs oculomotor systems), and despite the differences in the theoretical frameworks used to interpret the data, a consistent theme emerging from all studies of SAT is that it involves adjusting the baseline and gain of neural processing.

At commitment time, decision-related activity tuned to the preferred target reached a peak that was the same in both blocks, at least at the population level (Fig. 9). This supports the idea that commitment occurs when the total intensity of neural activity related to a choice reaches a fixed threshold or dynamic attractor that is consistent across conditions (Hanes and Schall, 1996; Gold and Shadlen, 2007), in agreement with fMRI results in the preSMA (Ivanoff et al., 2008). Likewise, Hanks et al. (2014) found that lateral intraparietal area activity just before the saccade was similar for both "speed" and "accuracy" conditions (even though recordings were performed on different days). In contrast, Heitz and Schall (2012) found that activity in FEF movement neurons was lower when animals were instructed to respond more slowly, and suggested that this may be due to the properties of cells downstream of FEF.

Although the activity of decision-related cells around the time of commitment was similar between the slow and fast blocks (Fig. 9), we found that within each block it was correlated with DD in $30 \%$ of cells in PMd and M1 (Fig. 10 D,F). In particular, for those neurons that were positively correlated with $\mathrm{DD}$, the regression had a higher intercept and lower slope in the fast than the slow block (Figs. 10F, 11D,E); and for neurons negatively correlated with duration, the reverse was seen (Fig. $11 I, J$ ). This pattern of results is consistent with the block-dependent urgency signals estimated from behavioral data (Fig. 3B). We also found a similar pattern of results in the premovement activity of cells that correlate with the speed of movement (Fig. 12), suggesting that the same urgency signal energizes both the speed of a decision and the vigor of the selected movement (Thura et al., 2014). However, because of the low number of cells and trials, this conclusion must be considered preliminary.

It is possible that our results are specific to free-response tasks in which sensory information is changing, such as during natural 
behavior, but not to the kind of static perceptual discrimination tasks usually studied in the laboratory. However, the UGM provides a good fit to such data as well; and indeed, Hawkins et al. (2015a) showed that it fits better than perfect accumulator models without urgency to the classic data from Roitman and Shadlen (2002). Furthermore, Carland et al. (2015) showed that, even during random-dot motion discrimination tasks, the motion signal is not integrated with the long time constant usually assumed by EAMs, but with a time constant on the order of $200 \mathrm{~ms}$. This is consistent with the UGM and with the proposal that neural activity buildup is primarily caused by an urgency signal. Thus, it remains to be seen whether the UGM applies in general or whether there is sufficient justification for proposing a system for changing the mechanism in a task-dependent manner. Nevertheless, as noted above, the widespread finding of baseline and gain changes suggests a strategy for SAT adjustment that generalizes across many scenarios.

If an urgency signal controls the SAT, then what is its source? Previous work implicates the striatum (Forstmann et al., 2008; van Veen et al., 2008; Bogacz et al., 2010a; Nagano-Saito et al., 2012), which projects to PMd and M1 through the globus pallidus and dorsomedial thalamus (Middleton and Strick, 2000). Furthermore, there is compelling evidence that pallidal output regulates movement vigor (Horak and Anderson, 1984a, b; Turner and Desmurget, 2010). Because we observed a strong link between the vigor of movements and the urgency signal derived from choice behavior (Thura et al., 2014), we hypothesize that SATs for deciding and acting may be controlled by a shared urgency/vigor signal that originates in the basal ganglia and controls both the gain of PMd/M1 decision-related cells as well as the premovement burst of cells that influence movement velocity. This leads to the prediction that, while monkeys perform the tokens task, neural activity in the globus pallidus will exhibit patterns resembling our hypothetical urgency signal (Fig. 3B). Namely, it will not be directionally tuned during deliberation but will be related to elapsing time and will change when monkeys switch between a hasty versus a conservative decision policy.

\section{References}

Balci F, Simen P, Niyogi R, Saxe A, Hughes JA, Holmes P, Cohen JD (2011) Acquisition of decision making criteria: reward rate ultimately beats accuracy. Atten Percept Psychophys 73:640-657. CrossRef Medline

Bogacz R, Wagenmakers EJ, Forstmann BU, Nieuwenhuis S (2010a) The neural basis of the speed-accuracy tradeoff. Trends Neurosci 33:10-16. CrossRef Medline

Bogacz R, Hu PT, Holmes PJ, Cohen JD (2010b) Do humans produce the speed-accuracy trade-off that maximizes reward rate? Q J Exp Psychol (Hove) 63:863-891. CrossRef Medline

Britten KH, Shadlen MN, Newsome WT, Movshon JA (1992) The analysis of visual motion: a comparison of neuronal and psychophysical performance. J Neurosci 12:4745-4765. Medline

Brunton BW, Botvinick MM, Brody CD (2013) Rats and humans can optimally accumulate evidence for decision-making. Science 340:95-98. CrossRef Medline

Carland MA, Marcos E, Thura D, Cisek P (2015) Evidence against perfect integration of sensory information during perceptual decision-making. J Neurophysiol. Advance online publication. doi:10.1152/jn.00264.2015. CrossRef Medline

Chittka L, Skorupski P, Raine NE (2009) Speed-accuracy tradeoffs in animal decision making. Trends Ecol Evol 24:400-407. CrossRef Medline

Churchland AK, Kiani R, Shadlen MN (2008) Decision-making with multiple alternatives. Nat Neurosci 11:693-702. CrossRef Medline

Churchland MM, Santhanam G, Shenoy KV (2006) Preparatory activity in premotor and motor cortex reflects the speed of the upcoming reach. J Neurophysiol 96:3130-3146. CrossRef Medline

Cisek P (2007) Cortical mechanisms of action selection: the affordance compe- tition hypothesis. Philos Trans R Soc Lond B Biol Sci 362:1585-1599. CrossRef Medline

Cisek P, Puskas GA, El-Murr S (2009) Decisions in changing conditions: the urgency-gating model. J Neurosci 29:11560-11571. CrossRef Medline

Ditterich J (2006) Evidence for time-variant decision making. Eur J Neurosci 24:3628-3641. CrossRef Medline

Drugowitsch J, Moreno-Bote R, Churchland AK, Shadlen MN, Pouget A (2012) The cost of accumulating evidence in perceptual decision making. J Neurosci 32:3612-3628. CrossRef Medline

Forstmann BU, Dutilh G, Brown S, Neumann J, von Cramon DY, Ridderinkhof KR, Wagenmakers EJ (2008) Striatum and pre-SMA facilitate decision-making under time pressure. Proc Natl Acad Sci U S A 105: 17538-17542. CrossRef Medline

Gluth S, Rieskamp J, Büchel C (2012) Deciding when to decide: timevariant sequential sampling models explain the emergence of value-based decisions in the human brain. J Neurosci 32:10686-10698. CrossRef Medline

Gold JI, Shadlen MN (2007) The neural basis of decision making. Annu Rev Neurosci 30:535-574. CrossRef Medline

Green DM, Swets JA (1966) Signal detection theory and psychophysics. New York: Wiley.

Grossberg S (1973) Contour enhancement, short term memory, and constancies in reverberating neural networks. Studies Appl Math 52:213-257. CrossRef

Hanes DP, Schall JD (1996) Neural control of voluntary movement initiation. Science 274:427-430. CrossRef Medline

Hanks T, Kiani R, Shadlen MN (2014) A neural mechanism of speedaccuracy tradeoff in macaque area LIP. Elife 3:e02260. CrossRef Medline

Hawkins GE, Wagenmakers EJ, Ratcliff R, Brown SD (2015a) Discriminating evidence accumulation from urgency signals in speeded decision making. J Neurophysiol 114:40-47. CrossRef Medline

Hawkins GE, Forstmann BU, Wagenmakers EJ, Ratcliff R, Brown SD (2015b) Revisiting the evidence for collapsing boundaries and urgency signals in perceptual decision-making. J Neurosci 35:2476-2484. CrossRef Medline

Heitz RP (2014) The speed-accuracy tradeoff: history, physiology, methodology, and behavior. Front Neurosci 8:150. CrossRef Medline

Heitz RP, Schall JD (2012) Neural mechanisms of speed-accuracy tradeoff. Neuron 76:616-628. CrossRef Medline

Horak FB, Anderson ME (1984a) Influence of globus pallidus on arm movements in monkeys: II. Effects of stimulation. J Neurophysiol 52: 305-322. Medline

Horak FB, Anderson ME (1984b) Influence of globus pallidus on arm movements in monkeys: I. Effects of kainic acid-induced lesions. J Neurophysiol 52:290-304. Medline

Ivanoff J, Branning P, Marois R (2008) fMRI evidence for a dual process account of the speed-accuracy tradeoff in decision-making. PLoS One 3:e2635. CrossRef Medline

Mansfield EL, Karayanidis F, Jamadar S, Heathcote A, Forstmann BU (2011) Adjustments of response threshold during task switching: a model-based functional magnetic resonance imaging study. J Neurosci 31:14688-14692. CrossRef Medline

Middleton FA, Strick PL (2000) Basal ganglia output and cognition: evidence from anatomical, behavioral, and clinical studies. Brain Cogn 42: 183-200. CrossRef Medline

Moran DW, Schwartz AB (1999) Motor cortical representation of speed and direction during reaching. J Neurophysiol 82:2676-2692. Medline

Nagano-Saito A, Cisek P, Perna AS, Shirdel FZ, Benkelfat C, Leyton M, Dagher A (2012) From anticipation to action, the role of dopamine in perceptual decision making: an fMRI-tyrosine depletion study. J Neurophysiol 108:501-512. CrossRef Medline

Ratcliff R (1978) A theory of memory retrieval. Psychol Rev 85:59-108. CrossRef

Ratcliff R, Hasegawa YT, Hasegawa RP, Smith PL, Segraves MA (2007) Dual diffusion model for single-cell recording data from the superior colliculus in a brightness-discrimination task. J Neurophysiol 97:1756-1774. Medline

Roitman JD, Shadlen MN (2002) Response of neurons in the lateral intraparietal area during a combined visual discrimination reaction time task. J Neurosci 22:9475-9489. Medline

Shadlen MN, Britten KH, Newsome WT, Movshon JA (1996) A computational analysis of the relationship between neuronal and behavioral responses to visual motion. J Neurosci 16:1486-1510. Medline 
Standage D, You H, Wang DH, Dorris MC (2011) Gain modulation by an urgency signal controls the speed-accuracy trade-off in a network model of a cortical decision circuit. Front Comput Neurosci 5:7. CrossRef Medline

Standage D, Blohm G, Dorris MC (2014) On the neural implementation of the speed-accuracy trade-off. Front Neurosci 8:236. CrossRef Medline

Thura D (2015) How to discriminate conclusively among different models of decision-making? J Neurophysiol. Advance online publication. doi:10.1152/jn.00911.2015. CrossRef Medline

Thura D, Cisek P (2011) Neural activity during adjustment of the speedaccuracy trade-off in a reach decision task. Soc Neurosci Abstr.

Thura D, Cisek P (2012) Neural bases of speed/accuracy trade-off adjustments during decision-making and movement execution in monkeys. Soc Neurosci Abstr.

Thura D, Cisek P (2014) Deliberation and commitment in the premotor and primary motor cortex during dynamic decision making. Neuron 81 : 1401-1416. CrossRef Medline

Thura D, Beauregard-Racine J, Fradet CW, Cisek P (2012) Decision making by urgency gating: theory and experimental support. J Neurophysiol 108: 2912-2930. CrossRef Medline
Thura D, Cos I, Trung J, Cisek P (2014) Context-dependent urgency influences speed-accuracy trade-offs in decision-making and movement execution. J Neurosci 34:16442-16454. CrossRef Medline

Turner RS, Desmurget M (2010) Basal ganglia contributions to motor control: a vigorous tutor. Curr Opin Neurobiol 20:704-716. CrossRef Medline

Usher M, McClelland JL (2001) The time course of perceptual choice: the leaky, competing accumulator model. Psychol Rev 108:550-592. CrossRef Medline

van Veen V, Krug MK, Carter CS (2008) The neural and computational basis of controlled speed-accuracy tradeoff during task performance. J Cogn Neurosci 20:1952-1965. CrossRef Medline

Wang XJ (2002) Probabilistic decision making by slow reverberation in cortical circuits. Neuron 36:955-968. CrossRef Medline

Wenzlaff H, Bauer M, Maess B, Heekeren HR (2011) Neural characterization of the speed-accuracy tradeoff in a perceptual decision-making task. J Neurosci 31:1254-1266. CrossRef Medline

Yang T, Shadlen MN (2007) Probabilistic reasoning by neurons. Nature 447:1075-1080. CrossRef Medline 